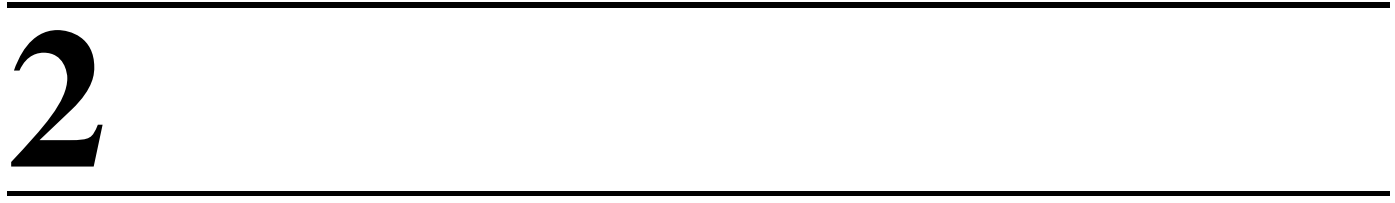

\title{
FUNDAMENTALS OF POLYMER NANOCOMPOSITE TECHNOLOGY
}

\author{
E. Manias, G. Polizos, H. Nakajima, and M. J. Heidecker \\ Pennsylvania State University, University Park, Pennsylvania
}

\subsection{INTRODUCTION}

The term nanocomposite is widely employed to describe an extremely broad range of materials, where one of the components has a dimension on the submicron scale. A better and far more restrictive definition would require that a true nanocomposite be a fundamentally new material (hybrid) in which the nanometerscale component or structure gives rise to intrinsically new properties, which are not present in the respective macroscopic composites or the pure components. The latter definition necessitates that the nanostructure has dimensions smaller than a characteristic scale that underlies a physical property of the material. For example, for the electronic properties of a conductor or semiconductor, this scale would relate to the de Broglie wavelength of the electron (ranging from a few nanometers for a metal to hundreds of nanometers for a semiconductor), for the mechanical properties of a polymer it would relate to the size of the polymer coil or crystal (again ranging from a few nanometers to hundreds of nanometers), and for the thermodynamic properties of a polymer glass it would relate to the cooperativity length (a few nanometers).

In this chapter we restrict our discussion even further, focusing on one subclass of polymer-inorganic nanocomposites, where the polymers are typically thermoplastics and the inorganic component is a high aspect ratio nanoscale filler. Particular emphasis will be given to principles that apply to pseudo-twodimensional layered inorganic fillers (such as 2:1 aluminosilicates, ${ }^{1-9}$ from where

Flame Retardant Polymer Nanocomposites, edited by Alexander B. Morgan and Charles A. Wilkie Copyright (c) 2007 John Wiley \& Sons, Inc. 
most of our examples will be drawn, and layered double hydroxides ${ }^{10}$ ), and to a smaller extent to pseudo-one-dimensional fillers (such as carbon nanotubes ${ }^{11}$ ). In these systems, concurrent improvements across multiple properties are typically achieved - with simultaneous enhancement of the mechanical, thermal, and thermomechanical response - in addition to new properties - such as improved barrier, flammability, and biodegradability behaviors-compared to the unfilled polymer. Consequently, the resulting nanocomposite material is better described by the term hybrid (denoting large-scale changes in multiple material characters) rather than polymer composite (a term traditionally associated with an incremental improvement in one or two key properties ${ }^{12-14}$ ).

For these nanocomposite systems, the fundamentally new properties typically originate from the change in the polymer nature in the vicinity of the filler, such as polymers adsorbed on filler surfaces or confined in between fillers, and as such, they depend strongly on the effective surface area of the fillers (i.e., the surface area of a single filler when completely dispersed or the surface area of the typical filler cluster). Thus, good dispersions of fillers would result in a true nanocomposite at rather low filler loadings, close to the percolation threshold of these high aspect ratio fillers (cf. below $3 \mathrm{vol} \%$ for typical layered silicates ${ }^{15}$ or 1 vol\% for single-walled nanotubes ${ }^{16}$ ). On the other hand, in the absence of dispersion, neither the nanometer-scale geometry of these fillers nor their ultrahigh surface area is exploited, and the resulting composite falls into the class of conventional composites despite the nanometer size of the individual inorganic fillers.

In the case of nanometer-thin layered inorganic fillers, it has long been known that polymers can effectively disperse clay minerals when the minerals are appropriately modified. ${ }^{1,2}$ The field has recently gained considerable momentum, due mainly to two major findings that pioneered the revival of these materials: First was the report of a nylon-6/montmorillonite (MMT) material from Unitika and Toyota researchers, ${ }^{17,18}$ where very moderate inorganic loadings resulted in concurrent and remarkable enhancements of thermal and mechanical properties. Second, Giannelis et al. found that it is possible to melt-mix polymers with clays without the use of organic solvents. ${ }^{19}$ Since then, the high promise for industrial applications has motivated vigorous research, which revealed concurrent dramatic enhancements in polymers by the dispersion of various nanometer-thin inorganic layered fillers. ${ }^{10,20-23}$ Where the property enhancements originate from the nanocomposite structure, these improvements are generally applicable across a wide range of polymers. ${ }^{6,10}$

In contrast, carbon nanotubes were discovered much more recently, first observed by Iijima, ${ }^{24}$ and since then they have been the focus of considerable research activity. This pseudo-one-dimensional form of carbon has remarkable physical and mechanical properties, such as structure-tunable electronic properties, ultrahigh thermal conductivity, and unmatched mechanical properties (e.g., stiffness, strength, and resilience). These characteristics, combined with recent advances enabling high-volume production of multi- and single-walled 
nanotubes, offer tremendous opportunities for the development of ultrahighperformance nanotube-reinforced nanocomposite materials. ${ }^{11}$

At this point we should also mention that this chapter is not intended to provide an extensive review of the polymer nanocomposites field. The reader interested in such reviews can refer to a number of related books, ${ }^{1-5}$ numerous compilations of relevant symposia and conference proceedings, and recent review articles. ${ }^{6-8,10,11}$ This chapter is, rather, an attempt toward a brief eclectic overview of topics highlighting the fundamentals that underlie the materials discussed in the remainder of the book.

\subsection{FUNDAMENTALS OF POLYMER NANOCOMPOSITES}

\subsubsection{Thermodynamics of Nanoscale Filler Dispersion}

As for polymer blends, the thermodynamics of mixing for polymers and nanofillers can be described through a balance of entropic and enthalpic factors, which determines whether a pristine or organically modified filler will be dispersed in a polymer. ${ }^{25-27}$ Especially for nanoparticles, favorable thermodynamics of mixing are essential since these ultrasmall particles are held together with very high apparent attractive forces [cf. eq. (2.3)] when immersed in liquid or polymeric media, and purely mechanical methods of mixing are not expected to be effective. Moreover, given the extensive amount of surface area that imposes entropic penalties for adsorbed, physisorbed, or intercalated macromolecules, the dispersion of nanofillers necessitates sufficiently favorable enthalpic contributions to overcome the entropic penalties.

For example, following the interfacial tension formalization of van OssChaudhury-Good, ${ }^{28}$ we consider two flat filler [e.g., layered silicate $(s)$ ] layers separated by an organic layer [e.g., alkyl surfactant film $(a)$ or an intercalated polymer film]. In this case, successive layers are held together with an adhesive energy:

$$
\Delta F_{\text {sas }}=-2 \gamma_{s a}=-2\left(\sqrt{\gamma_{s}^{\mathrm{LW}}}-\sqrt{\gamma_{a}^{\mathrm{LW}}}\right)^{2}-4\left(\sqrt{\gamma_{s}^{+}}-\sqrt{\gamma_{a}^{+}}\right)\left(\sqrt{\gamma_{s}^{-}}-\sqrt{\gamma_{a}^{-}}\right)
$$

when assuming additivity of apolar [Lifschitz-van der Waals (LW)] and polar [electron donor-acceptor, or Lewis acid-base (AB)] interaction terms, ${ }^{28}$ and using standard geometric combination rules:

$$
\gamma_{i j}=\gamma_{i j}^{\mathrm{LW}}+\gamma_{i j}^{\mathrm{AB}} \quad \text { with }\left\{\begin{array}{l}
\gamma_{i j}^{\mathrm{LW}} \simeq\left(\sqrt{\gamma_{i}^{\mathrm{LW}}}-\sqrt{\gamma_{j}^{\mathrm{LW}}}\right)^{2} \\
\gamma_{i j}^{\mathrm{AB}} \simeq 2\left(\sqrt{\gamma_{i}^{+}}-\sqrt{\gamma_{j}^{+}}\right)\left(\sqrt{\gamma_{i}^{-}}-\sqrt{\gamma_{j}^{-}}\right)
\end{array}\right.
$$

The $i$ and $j$ subscripts correspond to the various system components (layered silicate $s$, alkyl surfactant film $a$, and polymer $p$ ) and the LW and AB superscripts to the nature of interactions (apolar LW and polar AB). These relations can be 
converted into the Hamaker constant formalization by setting $\gamma_{i}^{\mathrm{LW}}=A_{i} / 24 \pi l_{o}^{2}$ with $l_{o}=1.58 \AA$. In the case of $2: 1$ aluminosilicates organically modified by alkyl surfactants, the attractive interaction energy of eq. (2.1) would correspond (Figure 2.1) to an adhesive pressure between the parallel flat surfaces of

$$
P=\frac{A}{6 \pi d^{3}}=\frac{-12 \pi l_{o}^{2} \Delta F_{\text {sas }}}{6 \pi d^{3}}
$$

where $d$ is the thickness of the organic interlayer film. Given that typical alkyl surfactant modifications - butyl to dioctadecyl — correspond to a surfactant layer thickness of 0.5 to $1 \mathrm{~nm}$, the corresponding adhesive pressure between successive silicate layers is at least ${ }^{32} 10^{5}$ to $10^{4}$ bar (cf. Figure 2.1). Thus, favorable enthalpic interactions are absolutely necessary for filler dispersion and nanocomposite formation.

Focusing on polymer nanocomposites based on organically modified layered silicates, Vaia et al. published a tractable approach to calculate the entropic and enthalpic contributions to the free energy of mixing ${ }^{25}$ and have used this to predict miscibility of polystyrene with alkylammonium-modified silicates ${ }^{26}$ (montmorillonite and fluorohectorite). According to this model, the entropic contributions are unfavorable and rather small: Specifically, the conformational entropy penalty of polymer confinement is compensated by an increase in conformational freedom of the tethered surfactants upon dispersion for gallery increases of up to $0.7 \mathrm{~nm}$, and adopts small unfavorable values for larger gallery increases (see Figure 4 of Vaia et al. ${ }^{25}$ ). Consequently, small per-monomer favorable enthalpic interactions

\begin{tabular}{llcc}
\hline Material & $\gamma^{L W}$ & $\gamma^{+}$ & $\gamma^{-}$ \\
\hline Water $^{29}$ & 21.8 & 25.5 & 25.5 \\
Montmorillonite $^{26}$ & 66 & 0.7 & 36 \\
Alkane $^{29}\left(\mathrm{C}_{12} \mathrm{C}_{18}\right)$ & 26 & 0 & 0 \\
Polypropylene $^{29}$ & 26 & 0 & 0 \\
Polyethylene $^{29}$ & 33 & 0 & 0 \\
Polystyrene $^{29}$ & 42 & 0 & 1.1 \\
PMMA $^{29}$ & 40.6 & 0 & 12 \\
Carbon nanotube $^{*}$ 30 & 18.4 & 12 & 12 \\
PET $^{31}$ & 43.5 & 0.01 & 6.8 \\
Nylon 6, 6 $^{29}$ & 36.4 & 0.02 & 21.6 \\
\hline
\end{tabular}

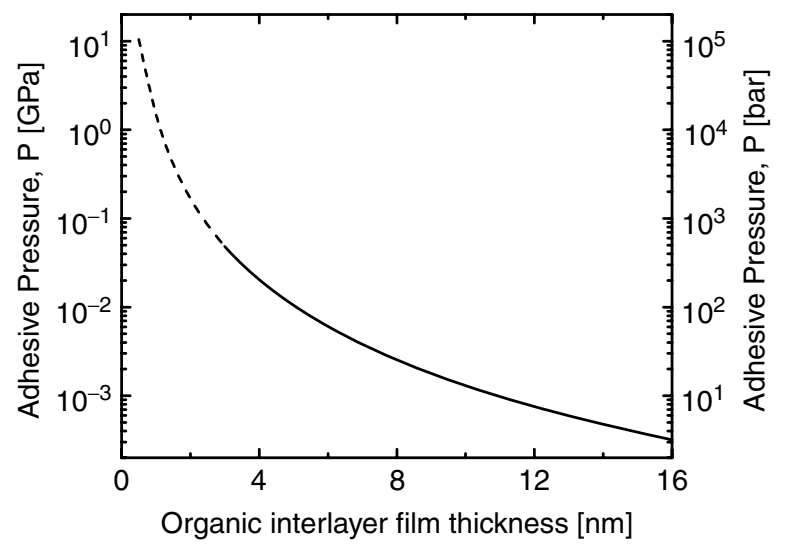

FIGURE 2.1 (Left) Various surface tension components $\gamma\left(\mathrm{mJ} / \mathrm{m}^{2}\right)$ for materials discussed in the text ( ${ }^{*}$ from $\gamma^{\mathrm{AB}} \simeq 24 \mathrm{~mJ} / \mathrm{m}^{2}$ assuming that $\gamma^{+} / \gamma^{-}=1$ ). (Right) Adhesive pressure versus interlayer thickness as predicted by eq. (2.3) for two flat montmorillonite surfaces separated by apolar organic films (e.g., an olefin). For small film thicknesses $(<2.5$ to $3 \mathrm{~nm})$ this continuum approach is not valid; rather, the adhesive pressure has discontinuous stable maxima ${ }^{32}$ (much higher than the dashed line) which correspond to integer numbers of monomer layers. 
can drive dispersion of these nanofillers in the polymer and promote the formation of a nanocomposite. These favorable enthalpic interactions are an excess enthalpy, akin to the $\chi$ parameter definition in Flory-Huggins theory; for silicate $(s)$ modified by a surfactant $(a)$ and a polymer $(p)$, this excess enthalpic interaction per area can be approximated ${ }^{25}$ by $\Delta H \sim \epsilon_{p s}+\epsilon_{p a}-\left(\epsilon_{a a}+\epsilon_{a s}\right)$, where $\epsilon_{i j}$ is a measurement of the pairwise interaction between components $i$ and $j$ [which can be quantified through pairwise atomic interaction parameters, cohesive energy densities, solubility parameters, or interfacial tension (Hamaker constants) formulations $\left.{ }^{29,32}\right]$. For most polymers and surfactants $\epsilon_{p a}-\epsilon_{a a} \ll \epsilon_{p s}-\epsilon_{a s}$, and to a first approximation for polymer/surfactant-modified inorganic nanocomposites, favorable enthalpy for mixing is achieved when the polymer-inorganic interactions are more favorable than the surfactant-inorganic interactions.

Following our prior nomenclature, dispersion would dictate a negative interaction energy change (upon mixing), which corresponds to a positive interfacial tension difference $\left(\gamma_{a s}-\gamma_{p s}\right)$. For an apolar $\left(\gamma_{a}^{ \pm} \simeq 0\right)$ alkyl surfactant (e.g., dodecane to nonadecane, ${ }^{29} \gamma_{a}^{\mathrm{LW}} \simeq 26 \mathrm{~mJ} / \mathrm{m}^{2}$ ) used to organically modify a typical silicate (e.g., montmorillonite, with ${ }^{26} \gamma_{s}^{\mathrm{LW}} \simeq 66 \mathrm{~mJ} / \mathrm{m}^{2}, \gamma_{s}^{+} \simeq 0.7 \mathrm{~mJ} / \mathrm{m}^{2}$, and $\left.\gamma_{s}^{-} \simeq 36 \mathrm{~mJ} / \mathrm{m}^{2}\right)$, miscibility would be achieved with any polymer for which

$$
\gamma_{\text {excess }}^{\text {total }}=\left(\sqrt{\gamma_{p}^{\mathrm{LW}}}-\sqrt{66}\right)^{2}+2\left(\sqrt{\gamma_{p}^{+}}-\sqrt{0.7}\right)\left(\sqrt{\gamma_{p}^{-}}-\sqrt{36}\right)-9.1 \mathrm{~mJ} / \mathrm{m}^{2}<0
$$

This is satisfied for most polymers (e.g., Table XIII-5 in van Oss' book ${ }^{29}$ ) except perfluorinated polymers and most of the polyolefins (polypropylene, polyisobutylene, etc). Miscibility is also promoted for all apolar polymers $\left(\gamma_{p}^{ \pm} \simeq 0\right)$ with $26 \mathrm{~mJ} / \mathrm{m}^{2}<\gamma_{p}^{\mathrm{LW}}<125 \mathrm{~mJ} / \mathrm{m}^{2}$, and for polar polymers with $\gamma_{p s}^{\mathrm{LW}} \simeq 26 \mathrm{~mJ} / \mathrm{m}^{2}$ and $\gamma_{p s}^{\mathrm{AB}}<0$ (i.e., as Vaia ${ }^{26}$ states, $\gamma_{p}^{+}>0.7 \mathrm{~mJ} / \mathrm{m}^{2}$ and $\gamma_{p}^{-}<36 \mathrm{~mJ} / \mathrm{m}^{2}$, or $\gamma_{p}^{+}<0.7 \mathrm{~mJ} / \mathrm{m}^{2}$ and $\gamma_{p}^{-}>36 \mathrm{~mJ} / \mathrm{m}^{2}$ ). Thus, for most polymers the commonly used organic modification by alkyl-cationic surfactants is adequate to create sufficient excess enthalpy and promote nanocomposite formation with montmorillonite.

In a different approach, 27 a longer macromolecular "surfactant" that would increase the layer separation to 5 to $10 \mathrm{~nm}$ necessitates much smaller favorable enthalpic contributions since the adhesive pressure to be overcome is about a thousand times smaller. This last theoretical prediction has been verified for polypropylene $(\mathrm{PP})^{33}$ in the absence of excess enthalpic interactions (i.e., $\gamma_{\mathrm{PP}}^{\mathrm{LW}}=26 \mathrm{~mJ} / \mathrm{m}^{2} \simeq$ $\gamma_{a}^{\mathrm{LW}}$ and $\gamma_{\mathrm{PP}}^{ \pm}=0$, and eq. (2.4) yields $\gamma_{\text {excess }}^{\text {total }} \simeq 0$ ), which in turn implies that for short surfactants the entropic penalties from the physisorbed PP will hinder spontaneous miscibility, whereas the entropic gains from longer surfactants would promote miscibility. ${ }^{27}$ At this point, we would like to make three more comments:

1. It should be obvious that free energy calculations cannot be done on a per molecule basis, but rather, the free energy of the system or the free energy per volume must be calculated. Thus, certain parameters that were omitted herein [such as the monomeric volumes of polymer and surfactant and the 
grafting density of the surfactant on the filler-in the case of silicates this would be proportional to the cation exchange capacity (CEC)] must also enter the calculations. ${ }^{26}$ The arguments above [e.g., eq. (2.4)] can be used when there is a substantial fraction of both polymer and surfactant in contact with the filler surface: for example, in the case of 2:1 aluminosilicates $0.65<\mathrm{CEC}<1.7 \mathrm{meq} / \mathrm{g}$ (or equivalently, surfactant grafting densities of one surfactant per $2<A<0.8 \mathrm{~nm}^{2}$ ), and still provide only approximate values or criteria. A more detailed discussion is provided elsewhere. ${ }^{26}$

2. In the case of polypropylene (PP), the approach described above yields a zero excess enthalpic interaction for an alkyl-modified silicate [since ${ }^{29}$ $\gamma_{\mathrm{PP}}^{\mathrm{LW}}=25.7 \mathrm{~mJ} / \mathrm{m}^{2} \simeq \gamma_{a}^{\mathrm{LW}}$ and $\gamma_{\mathrm{PP}}^{ \pm} \simeq 0$, eq. (2.4) yields $\left.\gamma_{\mathrm{excess}}^{\text {total }} \simeq 0\right]$, which implies that the entropic factors, albeit small in magnitude, will hinder spontaneous miscibility.

3. Under the approximations and assumptions mentioned above and without considering any entropic contributions, the interfacial (adhesive) energy per area of a polymer and a silicate is given by ${ }^{28}$

$$
\Delta F_{p s}^{\mathrm{total}}=\Delta F_{p s}^{\mathrm{LW}}+\Delta F_{p s}^{\mathrm{AB}}=\left(\gamma_{p s}^{\mathrm{LW}}-\gamma_{p}^{\mathrm{LW}}-\gamma_{s}^{\mathrm{LW}}\right)+\left(\gamma_{p s}^{\mathrm{AB}}-\gamma_{p}^{\mathrm{AB}}-\gamma_{s}^{\mathrm{AB}}\right)
$$

Substituting $\gamma_{p s}^{\mathrm{LW}}$ and $\gamma_{p s}^{\mathrm{AB}}$ from eq. (2.2) yields

$$
\Delta F_{p s}^{\mathrm{total}}=-2 \sqrt{\gamma_{p}^{\mathrm{LW}} \gamma_{s}^{\mathrm{LW}}}-2\left(\sqrt{\gamma_{p}^{+} \gamma_{s}^{-}}+\sqrt{\gamma_{p}^{-} \gamma_{s}^{+}}\right)
$$

which for a strictly apolar polymer becomes

$$
\Delta F_{p s}^{\mathrm{total}}=-2 \sqrt{\gamma_{p}^{\mathrm{LW}} \gamma_{s}^{\mathrm{LW}}}
$$

\subsubsection{Synthetic Routes for Nanocomposite Formation}

For traditional composite materials, high performance requires, in a first approach, homogeneous and thermodynamically stable dispersion of the fillers in the polymer matrix. To this end, the two major hurdles to be overcome are (1) deaggregation of the filler assemblies (clusters of fillers often containing tens, hundreds, or even millions of filler particles, associated with very strong interparticle forces ${ }^{32}$ ), and (2) achieving sufficiently strong polymer-filler interfaces, required for good mechanical coupling between the matrix and the filler. Both these requirements are also necessary in polymer-based nanocomposites, and depending on the nanofiller, there exist additional hurdles that need to be overcome toward nanocomposite formation. Examples of such challenges include entropic effects of polymers in nanoscopic confinements between two-dimensional fillers, as discussed in Section 2.2.1; deaggregation of intertwined one-dimensional filler clusters, as in carbon nanotube bundles or ropes; and overcoming the much faster kinetics (compared with colloidal micrometer-sized fillers) of nanofiller reaggregation. 
As for the thermodynamic consideration in Section 2.2.1, we attempt to highlight these challenges by describing in some detail the most common synthetic routes for nanocomposite formation employed for polymer/layeredinorganic hybrids. Most examples are drawn from layered-silicate fillers, but the conclusions are general across most nanofillers, and one should be able to envision similar strategies for nanocomposite formation based on other types of nanofillers.

2.2.2.1 Solution-Aided Dispersion and Brute-Force Melt Processing In most cases, polymer-inorganic systems that do not possess favorable thermodynamics for nanocomposite formation can be "trapped" in dispersed-even exfoliatedstructures through solvent casting, sonication, or high-shear-rate/hightemperature extrusion. Such trapped structures are usually easy to achieve* but in most cases are neither thermodynamically stable nor amenable to further processing: for example, in Figure 2.2, x-ray diffraction (XRD) of precipitated PP-MMT hybrids from a co-suspension of polypropylene and o-MMT (organically modified montmorillonite) in trichlorobenzene (similar structures can be obtained from aggressive melt processing, such as high-shear-rate extrusion, ${ }^{34-37}$ or dynamic packing injection molding ${ }^{38}$ ).

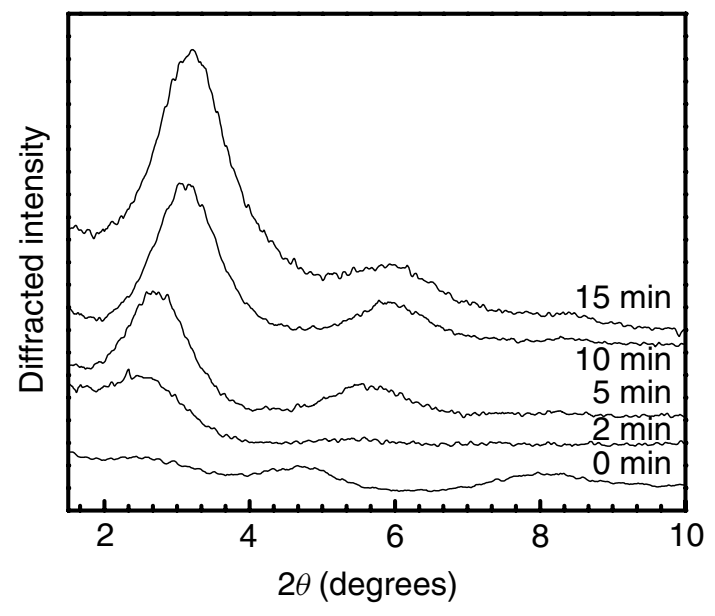

(a)

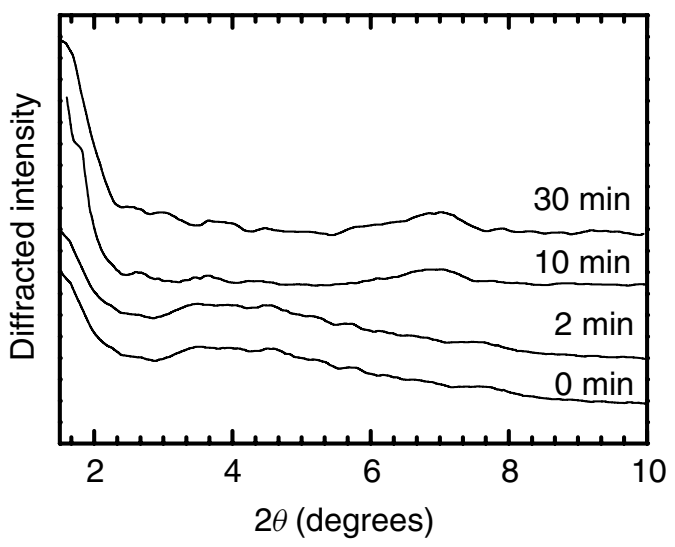

(b)

FIGURE 2.2 Structure evolution-stability of $(a)$ neat-PP/2C18-MMT and $(b)$ PP-gMA/2C18-MMT nanocomposites that were initially ( 0 min) trapped apart. XRD studies of compression-molded samples are shown. The neat-PP/2C18-MMT very fast collapses to intercalated-immiscible tactoids, whereas for the MA-functionalized PP, the trapped dispersed structure is maintained even under prolonged high-temperature processing. This suggests that the MA groups have sufficiently strong interactions with the MMT to prevent the polymer from sliding away from the inorganic layers. (Adapted from Ref. 49, copyright (c) 2001, American Chemical Society, with permission.)

*Easy or successful brute-force melt processing in the case of layered inorganic fillers is obviously limited to fillers of relatively small lateral size, given the very strong adhesive forces per area between such particles (Figure 2.1). 
However, upon subsequent processing by compression molding (at $180^{\circ} \mathrm{C}, 15$ tons) of these hybrids, the polymer melts and the trapped hybrid structure relaxes toward a thermodynamically favorable state. If the o-MMT dispersion is not thermodynamically favorable, the layers will collapse in low $d$-spacing parallel stacks (e.g., neat-PP/dimethyldioctadecylammonium-MMT; Figure 2.2a) during high-temperature processing, leading to a conventionally filled "macro" composite. However, when there exists favorable free energy for o-MMT/polymer mixing, the exfoliated filler structures are retained [e.g., polypropylene containing maleic anhydride (MA) functional groups and dimethyldioctadecylammonium MMT; Figure 2.2b]. Typically, this approach can yield stable dispersions only for polymers with strong specific interactions with MMT [e.g., polymers that hydrogen-bond to the silicates, such as poly(vinyl alcohol), ${ }^{39}$ polyurethanes, ${ }^{40,41}$ and polyamide- $\left.6^{42-44}\right]$. It is striking that only $0.5 \mathrm{~mol} \%$ of MA can have the same effect in PP. As expected, mechanical shear markedly reduces the time necessary for structure relaxation, and the structure of Figure $2.2 b$ is recovered after $8 \mathrm{~min}$ of mixing (extrusion at $180^{\circ} \mathrm{C}$ ). In concert, even after very moderate mixing $\left(1\right.$ to $3 \mathrm{~min}$ at $180^{\circ} \mathrm{C}$ ) trapped systems of neat-PP/2C18-MMT result in an immiscible or intercalated structure with a wide XRD reflection, extending from 1.8 to $2.7 \mathrm{~nm}$ in $d$-spacing. Along the same lines, when sonication is employed in polymer-nanofiller co-suspensions, instead of aggressive melt processing of the polymer with the nanofiller, similar trends can be observed and a well-dispersed structure can be stable when favorable interactions are present, as for example in polystyrene/imidazolium-montmorillonite systems. ${ }^{45}$ The sonication approach is, in general, a highly successful route for polymer nanocomposites based on carbon nanotubes, since the sonication can effectively disperse the nanotube bundles in solvent and subsequently in polymer matrix, and is commonly employed despite criticisms that sonication may cause tube breakdown.

This approach is qualitatively similar to the swelling agent approach, as for example, by Wolf et al. ${ }^{46}$ In such approaches an alkylammonium-exchanged montmorillonite is intercalated by an organic swelling agent such as ethylene glycol, naphtha, or heptane (all with boiling points below the processing or extrusion temperature). ${ }^{46}$ Subsequently, the swollen organo-modified clay is compounded with $\mathrm{PP}$ in a twin-screw extruder at $250^{\circ} \mathrm{C}$. At this processing temperature, the swelling agent evaporates, leading to the formation of a nanocomposite that is XRD silent. In principle, this is the same as the solution intercalation approach, where a solvent is employed to mix the o-MMT with the polymer, and a mostly exfoliated structure is trapped upon evaporation of the solvent. For fillers that cannot be surface-modified by grafted surfactants (such as graphite), the swelling agent approach is probably the most effective route for achieving filler dispersions.

In all the cases above and in the absence of polymer cross-linking or favorable thermodynamics to retain the dispersion achieved (by solvent, mechanical shear/vibration, swelling agent, etc.), the fillers will reaggregate upon further 
processing, and all the high-performance character due to nanoscale filler dispersion will be lost.

2.2.2.2 Static Melt Intercalation This method involves the mechanical mixing of a polymer with an appropriately modified filler and subsequent annealing above the softening temperature of the polymer. ${ }^{19}$ This approach provides the best route to test ${ }^{26}$ with sensitivity the thermodynamic arguments detailed above and to yield well-defined systems for fundamental studies. However, due to the quiescent processing conditions (absence of external shear), which eliminate any mechanical contribution for the dispersion of fillers, and to the very slow intercalation-exfoliation kinetics, ${ }^{47,48}$ such methods are typically very slow, thus having very limited applicability in industry.

We mention only one example for this method, polypropylene (PP) in organically modified montmorillonite (o-MMT), so as to elucidate how the thermodynamics of mixing can be tested. ${ }^{33,49}$ The challenge with PP is to design systems where the polymer-MMT interactions are more favorable than the surfactant-MMT interactions. As mentioned above, for an alkyl surfactant used as the organic modification in o-MMT, there is no excess enthalpy for mixing with $\mathrm{PP}\left(\gamma_{\text {excess }}^{\text {total }} \simeq 0\right)$, or in other words, the polymer-MMT interactions are equal to the surfactant-filler interactions. In agreement with the thermodynamic arguments presented above, minute amounts $(0.5$ to $1 \mathrm{~mol} \%)$ of randomly incorporated polar or polarizable $\left(\gamma^{\mathrm{AB}} \neq 0\right)$ functional groups, such as methylstyrene, hydroxyl, and maleic anhydride, can promote PP/o-MMT miscibility ${ }^{49}$ under static melt intercalation. Also, small blocks (1 to $5 \mathrm{~mol} \%$ ) of poly(methyl methacrylate) (PMMA) added to PP were shown to be sufficient to drive miscibility (in this case, $\gamma_{\mathrm{PMMA}}^{\mathrm{AB}}=0$, but $\gamma_{\mathrm{PMMA}}^{\mathrm{LW}} \simeq 40>\gamma_{a}^{\mathrm{LW}} \simeq 26 \mathrm{~mJ} / \mathrm{m}^{2}$ ), since the favorable thermodynamics for the PMMA can overcome the purely entropic barrier for the PP intercalation. Even in the extreme case, where the miscible block becomes as short as a single group, miscibility can still be achieved ${ }^{33}$ when this group possesses sufficiently strong interactions for the filler (as, e.g., an ammonium group ${ }^{33}$ ). On the other hand, if mixing is to be promoted for nonfunctionalized PP, a surfactant must be chosen with poorer interactions with the filler than the olefinic polymer (i.e., $\gamma^{\mathrm{AB}}=0$ and $\gamma^{\mathrm{LW}}<\gamma_{\mathrm{PP}}^{\mathrm{LW}} \simeq 26 \mathrm{~mJ} / \mathrm{m}^{2}$ ); such surfactants are, for instance, fluorinated or semifluorinated alkyls $\left(\gamma_{\mathrm{FE}}^{\mathrm{LW}} \simeq 18 \mathrm{~mJ} / \mathrm{m}^{2}\right)$. This strategy has also been proven experimentally. ${ }^{49}$

2.2.2.3 Melt Processing This is a very frequently used approach ${ }^{6,8,16}$ in which the polymer and the (usually organically modified) filler are incorporated together in a traditional polymer processing method, most commonly, extrusion or kneading, and less frequently, injection molding. In concert with the principles of static melt intercalation, favorable thermodynamics for mixing are introduced by the design of functionalities on the polymer and by the choice of the organic modification for the fillers. In addition to any thermodynamic contributions, mechanical shear provides a kinetic driving force for further dispersion of the fillers in the polymer matrix and accelerates substantially the kinetics of filler dispersion. 
The latter effect is particularly important for polymers that possess very high attractions for the filler surfaces and can be kinetically arrested under static melt intercalation. ${ }^{48}$ In many cases, end users of polymer nanocomposites are hesitant to incorporate nanofillers directly (in the form of ultrafine powders) in their current processing practices, and the concentrate or masterbatch two-step approach is preferred. In this case, first a polymer nanocomposite (concentrate) is formulated at relatively high filler loadings of about $25 \mathrm{wt} \%$, which can be processed and palletized to look like a normal polymer resin. This concentrate is subsequently diluted (i.e., let down) to the desired filler loading by pure polymer resin (cf. below).

2.2.2.4 Masterbatch Approaches Beyond any industrial reservations for incorporating nanoparticles directly into the final stages of processing, there also exist in some cases scientifically justified reasons to follow the concentrate or masterbatch approach. For example, in the first studies aiming to develop PP/o-MMT materials, ${ }^{34-37,46,50}$ polypropylene oligomers modified with either maleic anhydride (MA) or hydroxyl groups $(\mathrm{OH})$ were first mixed with octadecylammonium-exchanged montmorillonite, creating a masterbatch at high filler loadings which was subsequently blended with neat PP, usually assisted by strong mechanical shear in an extruder or mixer. In this way, the MA-polypropylene disperses the o-MMT, given the favorable thermodynamics, and in the second step PP and PP-g-MA are effectively at theta conditions, and the extrusion is promoting mixing due only to entropic reasons (cf. morphologies of miscible polymer blends). Although at first glance, this approach may seem similar to the one denoted above as "brute force," in the masterbatch case there do exist favorable thermodynamics for mixing, which not only result in more effective dispersions, but also stabilize the dispersed nanocomposite structure. However, the structure and properties of the resulting hybrid materials still depend strongly on the processing conditions, and in the case of PP, for example, they range from very moderate dispersions and property improvements ${ }^{34,36,37,46,50}$ to good dispersions and better-performing hybrids. ${ }^{35}$ Obviously, an MA-polypropylene pretreatment with very low maleic anhydride content does not promote nanocomposite formation, ${ }^{36}$ and very high maleic anhydride content makes the masterbatch so robust that MMT does not mix further with neat PP. ${ }^{34,37}$ Furthermore, the PP-g-MA can have marked effects on PP crystallization and, consequently, cause the mechanical properties to deteriorate, especially when the PP-g-MA is of substantially lower molecular weight or isotacticity than the PP matrix, or contains high levels of branching. Therefore, it is frequently necessary to develop several variants of a masterbatch [based on functional polymers with varied characteristics: e.g., in the case of PP with various molecular weights of PP-g-MA or in the case of polyethylene (PE) with various polymer microstructures (LDPE, LLDPE, HDPE, etc.)], depending on the specific characteristics of the polymer matrix for which they are intended.

2.2.2.5 In Situ Polymerization Schemes One of the cornerstone studies, and probably the single most important study in pioneering the revival of the polymer/ 
layered-silicate nanocomposites field, was the work by the Toyota group in which they polymerized polyamide- 6 in the presence of, and end-tethered on, the surfaces of montmorillonite layers. ${ }^{17,18}$ Since then, the strategy of in situ polymerization of a monomer in a co-suspension with inorganic filler has been employed successfully for a variety of polymers, with and without end tethering the macromolecules on the filler surfaces, and through various polymerization reactions, for a variety of polymers and fillers (detailed examples are discussed in a review article $^{8}$ ). In most cases, nanocomposites formed by in situ polymerization result in structures that are kinetically trapped (cf. the solution approach above) in a well-dispersed structure. In general, these structures do possess favorable thermodynamics to retain the filler dispersion upon subsequent processing (such as compression or injection molding of the hybrid after polymerization), since this method requires that the monomer initially disperses the inorganic particles sufficiently. However, if more polymer is added in the subsequent processing step (cf. an attempt to use the in situ polymerized hybrid as a masterbatch), in most cases there occurs a loss of the exfoliated structure achieved during the in situ polymerization step, and typically a less dispersed structure is obtained. For example, attempts to dilute the polyamide-6/montmorillonite nanocomposite ${ }^{17}$ with pure polyamide- 6 or an in situ poly $(\epsilon$-caprolactone $) /$ montmorillonite hybrid ${ }^{51,52}$ with pure poly( $\epsilon$-caprolactone $)$ result in collapse of the mostly exfoliated in situ structure. Typically, this well-dispersed in situ structure becomes intercalated upon addition of the homopolymer, where the inorganic fillers adopt a parallel stacking with a polymer bilayer (an intercalated layer about two monomers thin) in the interlayer gallery.

2.2.2.6 Extension to Other Fillers These ideas can be extended to other high aspect ratio fillers when taking their idiomophies into account. The ideas can be transferred almost as stated above to other two-dimensional and pseudo-twodimensional layered fillers [e.g., layered double hydroxide (LDH) ${ }^{10}$ or graphite] when addressing their differences from layered aluminosilicates; for example, LDH would require anionic surfactants, whereas graphite is not amenable to grafted modifications and an intercalated swelling agent is needed (cf. the masterbatch or solution approaches above).

For one-dimensional nanofillers, however, there are important differences that may necessitate different choices for nanocomposite formation. For example, in the case of carbon nanotubes, polymer-matrix nanocomposites can be fabricated using almost all of the schemes discussed above, but the effectiveness and importance of these schemes are very different from those of polymer/layeredinorganic nanocomposites. Dispersion of nanotubes is hindered not only by their high affinity for one another, but also by their ability to intertwine with one another, forming bundles or ropes. These often-large agglomerations are typically formed during synthesis of the nanotubes (especially for single-walled carbon nanotubes), which need to be well unbundled before attempting dispersion in a polymer matrix. At the same time, the reactive bonding of surfactants on the nanotube surfaces, although possible via multiple chemistries, ${ }^{53,54}$ most 
often causes deterioration of their remarkable physical properties (more so for single-walled than for multiwalled carbon nanotubes), in particular their thermal and electron conductivities, as well as their stiffness and strength.*

After these thoughts, and following our earlier discussion of polymer-silicate nanocomposites, it seems obvious that the nanocomposite formation schemes that depend on favorable thermodynamics (e.g., melt blending) or brute-force mechanical mixing are of limited use here, whereas the solution mixing and the in situ polymerization schemes should be much more effective. ${ }^{11,56}$ In fact, the most common approach for polymer-nanotube composite formation involves first unbundling the nanotube aggregates in solvent (most often aided by sonication and physisorbed surfactants, and centrifugal separation) and subsequent solution-aided dispersion in a polymer matrix. These solution-aided dispersions can effectively trap the nanotubes in a well-dispersed morphology after solvent evaporation (see, e.g., Refs. 57 and 58). Alternatively, instead of employing a physisorbed surfactant and two steps of solution dispersion, nanocomposites can be formed in a one-step solution process (much like their polymer-silicate counterparts, by co-dissolving the host polymers and nanotubes in a common solvent), as, for example, with poly(vinyl alcohol ${ }^{59}$ and polystyrene. ${ }^{16}$ For the same reason (i.e., employing 'solvent' to unbundle the nanotubes), in situ polymerization has also proven to be an effective method for producing well-dispersed nanocomposites. A characteristic example of this approach is the polymerization of PMMA in the presence of solution-dispersed nanotubes, leading to high-molecular-weight polymers and very good nanocomposite morphologies (see, e.g., Refs. 60-62).

Finally, unlike polymer/layered-silicate nanocomposites, melt processing is far less common for nanotube-reinforced nanocomposites. Melt processing relies on mechanical shear and thermodynamics to unbundle the nanotubes and disperse them further in a polymer matrix. Since neither of these two processes is expected to be very effective for ordinary polymers and nanotubes, typically the nanocomposites produced in this fashion have significant filler aggregation and comparably poor performance [e.g., high-density polyethylene, polypropylene, and polyamide-6/acrylonitrile-butadiene-styrene (ABS) have been melt-processed with nanotubes $\left.{ }^{63-65}\right]$. Since direct melt processing is inherently ineffective in dispersing nanotubes into polymers, melt processing will probably remain limited in practice except for those systems for which polymer-nanotube masterbatches can be developed at reasonable cost and with good nanotube dispersion.

\subsubsection{Dispersion Characterization: Common Techniques and Limitations}

Due to its ease of use and availability, simple Bragg-reflection powder x-ray diffraction is most commonly used to probe nanocomposite structure, especially for polymer/layered-inorganic filler hybrids where the $d_{001}$ basal reflection is

*This does not automatically imply that the respective nanocomposites are also characterized by deteriorated properties. For example, where good dispersions and/or covalent bonding occurs between the polymer matrix and the functionalized nanotubes, the nanocomposites can have very good mechanical property enhancements. ${ }^{55}$ 
indicative of filler-filler separation. However, the XRD can only detect the distance of periodically stacked layers; disordered (bunched together but not parallel stacked) or exfoliated layers are not detected, and large $d$-spacings (higher than $50 \mathrm{~nm}$ ) are sometimes not detectable by powder XRD. In general, for medium (ca. $1 \mu \mathrm{m}$ ) lateral size platelets, such as those in natural clays, even with favorable thermodynamics for nanocomposite formation, the structure is characterized by the coexistence of exfoliated, intercalated, and disordered layers. Thus, a silent XRD may hide a large number of disordered tactoids, whereas an XRD with an intercalated peak does not reveal the extent of exfoliation. In both cases, the nanocomposite properties are commonly affected dramatically by structures that are not manifested in the XRD, and thus XRD can be highly misleading when employed as a single tool for quantifying nanocomposite structure or even filler dispersion. Although detailed quantitative analysis of such XRD data ${ }^{66}$ in the low $2 \theta$ range, coupled with careful sample preparation and use of model reference samples, can yield substantially more information about the nanocomposite structure ${ }^{66}$ powder XRD is insufficient to capture and characterize the nanocomposite structure. Furthermore, when polymer-inorganic nanocomposites are based on fillers that are not two-dimensional in geometry (and thus do not have basal spacings, as for example carbon nanotubes and spherical or ellipsoidal nanoparticles), XRD is completely incapable of even a first-order qualitative determination of dispersion or structure.

Small-angle $\mathrm{x}$-ray scattering (SAXS) is probably the most informative widely available technique to characterize nanocomposite structure. The main hurdle with this method is converting the information collected in the $k$-space quantitatively into parameters that describe the real space morphology of the hybrids. As an example, for polymer/layered-inorganic fillers, simple ${ }^{67}$ and more realistic ${ }^{68}$ models of discoid scatters in organic matrices have been proposed that can be used to interpret scattering data into real space parameters for such nanocomposites. In a simple approach, ${ }^{67}$ after relatively simple analysis of the scattering data, average descriptors of the structure can be obtained which are of some value for quantifying the hybrid structure. A more complete description of structure necessitates much more careful design and implementation of scattering studies and more tedious analysis. ${ }^{68}$ Even where models for specific structures have been developed and methods for an experimental approach and analysis have been outlined, as for example in the case of layered inorganic nanoparticles, ${ }^{68}$ the amount of work involved to implement such approaches in real polymer nanocomposite systems has proven to be a barrier to the widespread use of SAXS as a common morphology characterization practice.

Transmission electron microscopy (TEM) is also widely employed, in its simplest bright-field mode, as a tool for direct visualization of the nanocomposite structure of polymer nanocomposites. This is possible because there exists sufficient contrast for the transmitted electrons between the polymer matrix and most fillers (inorganic particles, carbon in nanotubes or graphite, and almost all oxides) without polymer staining. In the extreme case, high-resolution TEM $^{69}$ can even provide a qualitative picture of the inorganic filler crystal structure, or can be 
combined with point electron diffraction to interrogate crystal structures in specific filler or polymer regions. Although TEM does not suffer from the same shortcomings as XRD, since it can visualize nanoscale fillers directly without the need for parallel stacking, it does have other limitations: First, it is very painstaking to obtain quantitative information about any of the characteristic parameters that describe the nanocomposite morphology. Such information can only be derived from image analyses of many and independent TEM images, so as to ensemble typical structures in the composite with some statistical importance. Second, since TEM is essentially a projection method, it is difficult to characterize structures normal to the large surface area of fillers; for example, almost all TEMs published for polymer/layered-silicate nanocomposites show images with the silicates positioned on the image edge-on, since layers parallel or oblique to the sample surface project as extended dark areas in a TEM image. Despite these limitations, we believe that informative TEMs should, at a minimum, complement XRD or other morphology studies, even if only to capture the hierarchical structures of the hybrid qualitatively at various length scales. Probably the additional information provided by TEMs is crucial when accompanying featureless XRD structures such as silent (no basal reflections) polymer/layered-nanofiller nanocomposites (which in most cases are wrongfully interpreted as exfoliated structures), polymer-nanotube hybrids, and polymer-nanoparticulate composites.

Finally, morphological information can also be obtained indirectly from methods that reflect the composite morphology into other macroscopic properties. Within the focus of this book, two examples of such methods can be mentioned: rheological measurements and cone calorimetry flammability methods. Both methods can sensitively detect well-dispersed nanofillers in a polymer matrix and can distinguish them from the respective conventional composites based on the same polymer and fillers but without nanometer-scale dispersion of the latter. We shall not provide further details on this; we just point the interested reader discussions of the cone calorimetry approach in subsequent chapters, and to a few representative references for the rheology. $7,16,70$

In summary, in lieu of providing a recipe for the characterization of nanocomposite morphology, we illustrate the limitations of the foregoing characterization techniques through an example of the plethora of parameters needed to describe the morphology of a polymer/layered-silicate nanocomposite (Figure 2.3). Even in this case, which can actually be interrogated by XRD characterization, only the distribution of basal (layer-layer distances within parallel stacked clusters) spacings can be obtained by XRD. SAXS can, in addition, provide some additional parameters ${ }^{67}$ such as mean number of layers per stack and "projected" lateral dimension of layers, while through more realistic models and analysis, ${ }^{68}$ only approximate values can be obtained for the rest of the important parameters (Figure 2.3). In almost all cases, a representative set of TEM structure observations should also be obtained (in addition to diffraction or scattering characterization), which should provide a qualitative description of structure, although there may be shortcomings in quantifying the various morphological parameters 


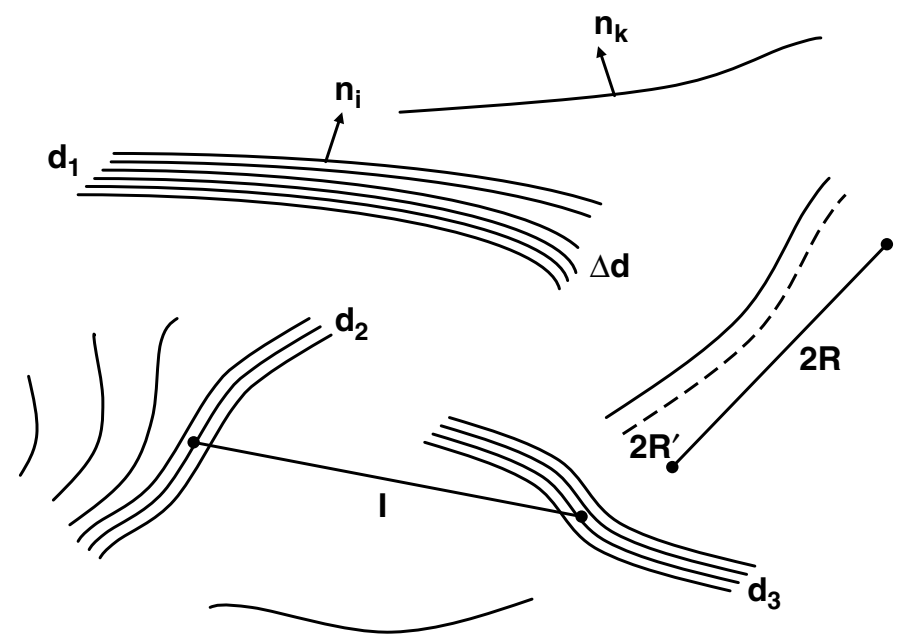

FIGURE 2.3 Relevant parameters needed to describe the morphology of a polymer/ layered-silicate nanocomposite. Layer parameters: layer thickness $(H)$, lateral contour size $\left(2 R^{\prime}\right)$, and corresponding projected lateral size $(2 R)$. Layer stack parameters: distribution of layer-layer distances within a stack $\left(d_{001}: d_{1}, d_{2}, d_{3}\right)$, distortion in $d(\Delta d)$, and mean number of layers per stack $(N)$. Distribution of stacks parameters: mean particleparticle distance between center of mass of stacks $(I)$, relative particle-particle orientation $\left[\phi\left(\vec{n}_{i}, \vec{n}_{k}\right)\right]$, and fraction of layer stacks consisting only of individual layers $(\chi)$. (Adapted from Ref. 68.)

(due to the local-only observation of morphologies, even by numerous TEM images).

\subsection{EFFECTS OF NANOFILLERS ON MATERIAL PROPERTIES}

\subsubsection{Effects on Polymer Crystallization}

2.3.1.1 Polymer-Specific Effects It is expected that the incorporation of nanoparticles in a semicrystalline polymer matrix would substantially affect the crystallization behavior of the polymer. Depending on polymer-filler interactions, three types of behavior can develop.

(a) Development of New Crystal Structures Where strong specific interactions exist between a filler and a polymer, a new crystal structure can develop in the vicinity of the filler, which is often not the same as the crystal structure of the unfilled polymer under normal crystallization conditions. The best example of such behavior is the case of polyamide-6/montmorillonite nanocomposites, in which the $\gamma$-crystal phase of polyamide is promoted next to the fillers. ${ }^{42-44}$ This behavior originates from the strong hydrogen bonding of the amide groups with the silicate $\left(\mathrm{SiO}_{x}\right)$ surfaces, and is, for the same reason, also observed in poly(vinyl alcohol)/MMT nanocomposites. ${ }^{39,71}$ A less frequent case, where new crystal structures are promoted by nanoscale fillers, also exists 
for polymers that develop nonbulk crystal phases when the polymer chains are aligned parallel to the filler's solid surfaces; two examples of such nanocomposites are polyvinyldene fluoride (PVDF) ${ }^{72}$ and syndiotactic polystyrene (sPS). ${ }^{73}$ In all cases where the inorganic surfaces promote growth of a different crystal phase, the nanocomposite mechanical and thermal properties can be enhanced through this mechanism when the surface-nucleated crystalline phase has better mechanical and thermal characteristics than those of the bulk crystal phase. Fillers with a large surface area maximize these filler-induced enhancements of the material properties; a dramatic manifestation of such a response is found in polyamide-6/montmorillonite nanocomposites.

(b) Polymer Amorphized by Filler In very few cases, such as poly(ethylene oxide) (PEO) $/ \mathrm{Na}^{+}-$montmorillonite nanocomposites, the polymer $-\mathrm{Na}^{+}$interactions are favorable to mixing but not conducive to crystallinity. ${ }^{74}$ Specifically, the crystallization of PEO nanocomposites based on alkali-cation bearing fillers is found to be inhibited, exhibiting a decrease in spherulite growth rate and crystallization temperature. Although the overall crystallization rate increases with silicate loading as a result of the extra nucleation sites that occur in the bulk PEO matrix (i.e., far from the silicate surfaces), PEO is highly amorphized near the montmorillonite surfaces. This behavior is attributed to the specific way that PEO interacts with $\mathrm{Na}^{+}$montmorillonite, where strong coordination of PEO to the surface $\mathrm{Na}^{+}$cations promotes noncrystalline (ether crown) PEO conformations.

(c) Heterogeneous Nucleation by Fillers For the vast majority of polymers, the effect of nanofillers on polymer crystallization relates only to crystal nucleation by the fillers (which typically increases proportionally to the number of individual filler clusters) and to changes in the kinetics of crystallization (which are typically characterized by a two- to fourfold decrease in the linear growth rate of crystallization). In these cases, and for filler loading below ca. $10 \mathrm{wt} \%$, the equilibrium melting temperature $\left(T_{m}^{0}\right)$ is not affected by the nanocomposite formation. For example, as shown in Figure 2.4 , the $T_{m}^{0}$ of PP-g-MA, PET, and PEO nanocomposites and the respective bulk polymers were estimated based on Hoffman-Weeks plots, and it is shown that moderate (below $10 \mathrm{wt} \%$ ) MMT addition does not change the $T_{m}^{0}$ value $\left[T_{m}^{0}(\mathrm{PP}-\mathrm{g}-\mathrm{MA})=183.8^{\circ} \mathrm{C}, T_{m}^{0}(\mathrm{PET})=\right.$ $260.1^{\circ} \mathrm{C}, T_{m}^{0}(\mathrm{PEO})=69.7^{\circ} \mathrm{C}$. These results are consistent with the literature reported earlier ${ }^{8}$ and make it possible to compare the crystallization kinetics of neat polymers and their nanocomposites at the same isothermal crystallization temperature. To further elucidate the effect of MMT on the crystallization kinetics of these polymers, isothermal crystallization measurements can be carried out with differential scanning calorimetry (DSC) and complemented by direct imaging of the crystallites [cross-polarization optical microscopy and atomic force microscopy (AFM)] for systems crystallized under the same conditions (Figure 2.4). Initially (crystals grow in three dimensions and the crystallites have not yet impinged), crystallization kinetics can be expressed as

$$
V_{f}^{c}=\frac{4}{3} \pi \rho_{n} G_{R}^{3} t^{3}
$$



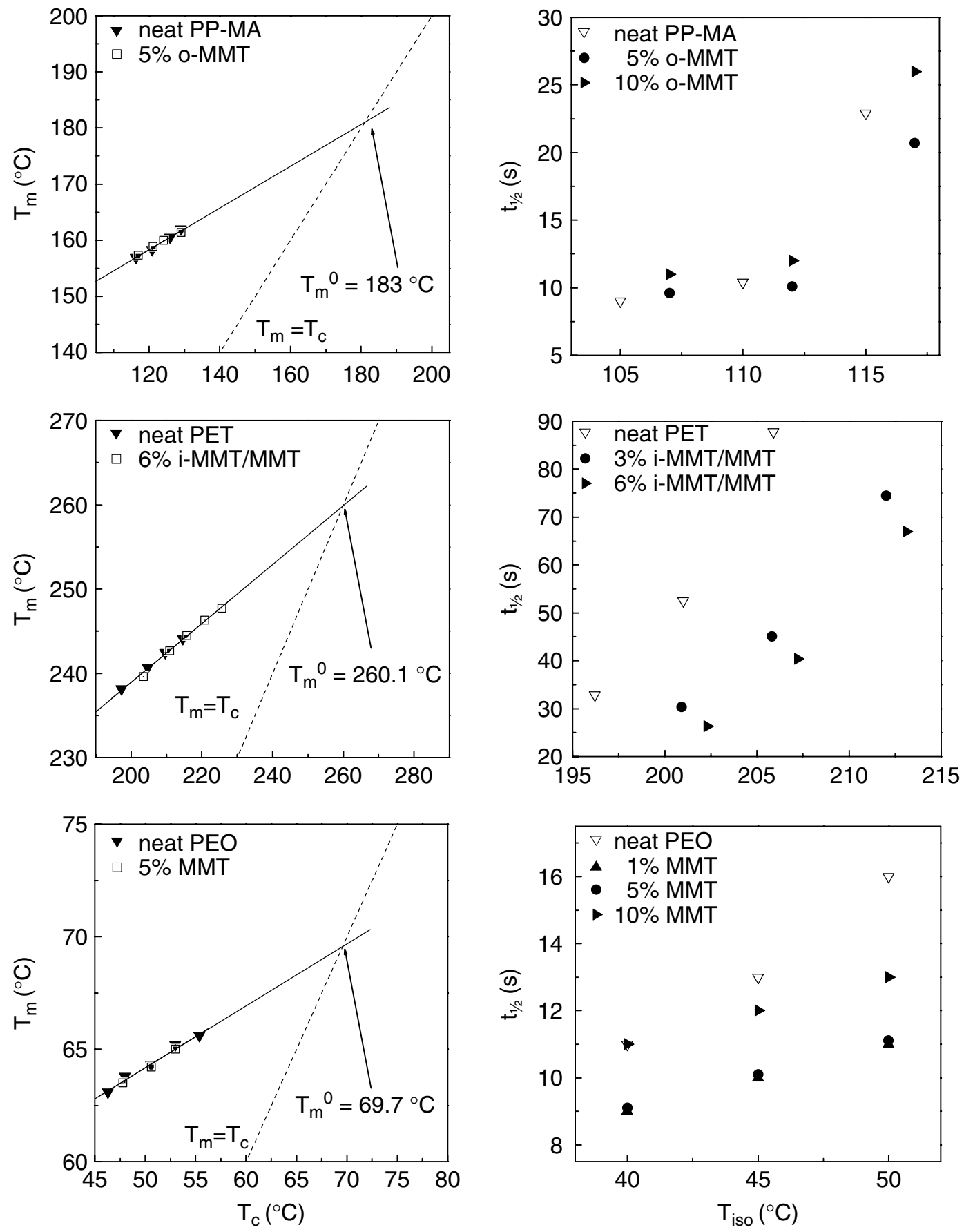

FIGURE 2.4 (Left) Hoffman-Weeks plots of neat polymers and their nanocomposites; the $T_{M}^{0}$ of the polymers is not affected by the nanocomposite formation. (Right) Half-time of crystallization for the same neat polymers and their nanocomposites; the overall crystallization rate is reduced for PET and PEO upon incorporation of an inorganic nanofiller, and is not affected for PP-g-MA. When accounting for changes in the nuclei density with filler incorporation, the linear growth rates $G_{R}$ are slowed down in all systems shown. For both panels: PP-g-MA (top), PET (middle), PEO (bottom). 
where $V_{f}^{c}$ is the total crystal volume (crystallinity), $\rho_{n}$ the nuclei density, $G_{R}$ the linear crystal growth rate, and $t$ the crystallization time. When $V_{f}^{c}$ is 0.5 , the corresponding crystallization time $t$ is defined as the half-time of crystallization $\left(t_{1 / 2}\right)$ and denotes the time necessary to reach $50 \%$ of the total enthalpy of crystallization under isothermal differential scanning calorimetry conditions (Figure 2.4). When the nuclei density, $\rho_{n}$, is measured by cross-polarized optical microscopy and/or AFM, the crystal linear growth rate can be estimated. The half-times of crystallization for neat polymers and their nanocomposites is shown in Figure 2.4 for various isothermal crystallization temperatures $\left(T_{\text {iso }}\right)$. As expected, the overall crystallization rate increases with clay or filler addition, as denoted by the decrease in $t_{1 / 2}$ upon the addition of nanofillers (this effect is rather small for PP-g-MA). However, accounting for the nuclei density increase in the nanocomposites (for PP-g-MA, the $\rho_{n}$ increased ca. six- to eightfold at 5 to $10 \mathrm{wt} \%$ o-MMT content; for PET, more than 500-fold at 3 to $6 \mathrm{wt} \%$ o-MMT; and for PEO, 20- to 50-fold for 5 to $10 \mathrm{wt} \%$ MMT), linear crystal growth is slowed down due to the introduction of clay across all systems. Despite the qualitative differences between PEO, PP, and PET crystallization when reinforced by MMT, and despite the quantitative differences in $t_{1 / 2}$, when the increase in nuclei density is accounted for, all systems show a $G_{R}$ decrease of 0.25 to 0.5 upon MMT addition (for PP the nanocomposite $G_{R}$ value is 0.5 of the bulk polymer value, for PET it is 0.25 , and for PEO it is 0.33 of the respective bulk polymers). This agreement between such different systems strongly indicates that the geometric constraints associated with the dispersion of MMT fillers is determining the effect (decrease) on the linear crystal growth rate in these systems rather than the polymer-MMT interactions. In the latter case, one would expect a qualitatively different effect in PET and PP compared to PEO, and also substantial quantitative differences between PET and PP. All these effects manifest themselves in differential scanning calorimetry studies, especially when the behavior of the neat (i.e., unfilled) polymer is compared against that of the respective nanocomposite (Figure 2.5).

2.3.1.2 General Effects Across Polymers Despite the variety of the nanofiller effects on polymer crystallinity, which originate from the various polymer-filler interactions, there also exist important common effects on the crystallinity due to the nanocomposite structure. The most important of these general effects is probably a general reduction in the size of the polymer crystallites upon nanocomposite formation. For example, in Figure 2.6 we compare the spherulites observed for unfilled polymers and their respective $3 \mathrm{wt} \%$ montmorillonite nanocomposites. Independent of how the fillers affect the nucleation and/or kinetics of crystallization, there is in all cases a substantial decrease in the spherulitic size. This behavior originates from the discontinuity of space caused by the inorganic fillers, which forces spherulites to have sizes comparable with the filler-filler separation, independent of the bulk polymer spherulite size. This effect is also independent of whether crystallization in the nanocomposite is nucleated homogeneously (PEO) or heterogeneously (PP, sPS), and of whether the fillers hinder crystallization 

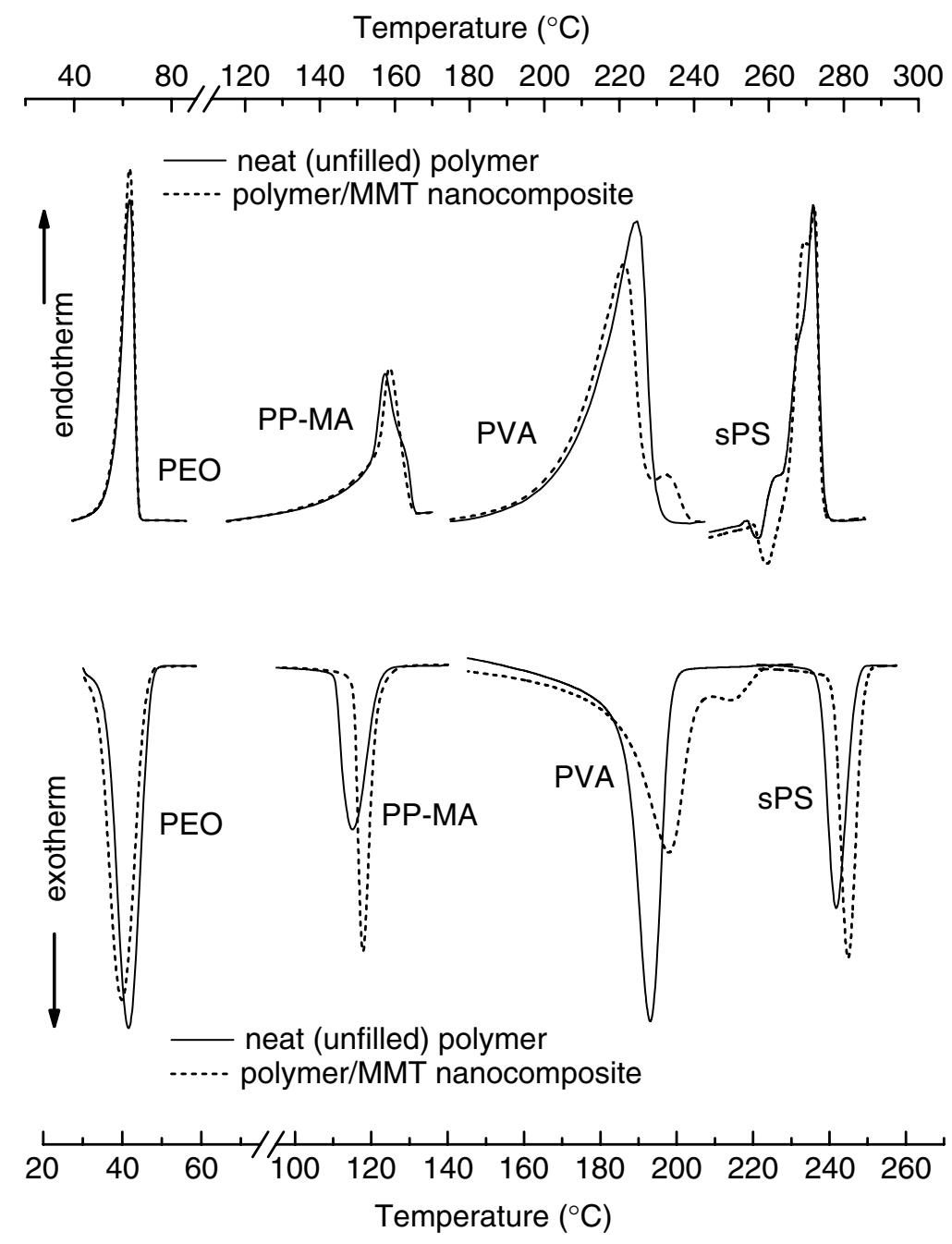

FIGURE 2.5 Differential scanning calorimetry comparison of unfilled polymers and their respective nanocomposites with montmorillonite layered silicates. (Top) Heating DSC scans: The crystalline melting point is markedly unaffected by the addition of fillers since the polymer crystal structure (e.g., the crystal unit cell) is not affected by the filler. A notable exception are those polymers where a new crystal structure is promoted near the filler surface, such as PVA, syndiotactic-PS, and polyamide (not shown here). (Bottom) Cooling DSC scans: The crystallization point is strongly affected by the fillers, bearing traces of heterogeneous nucleation (PP, sPS, PVA), crystallization of new crystal structures (PVA), or hindering of crystallization near a filler (PEO).

(PEO), promote new crystal structures (sPS), or simply act as heterogeneous nucleating agents (PP).

2.3.1.3 Effects of One-Dimensional Nanofillers Like layered-inorganic fillers, carbon nanotubes influence polymer crystallization when incorporated as filler in the polymer matrix; however, these effects do not have as wide a variety as the layered silicates discussed above. In the vast majority of reports, carbon nanotubes act simply as heterogeneous nucleating agents in crystallizable polymer 


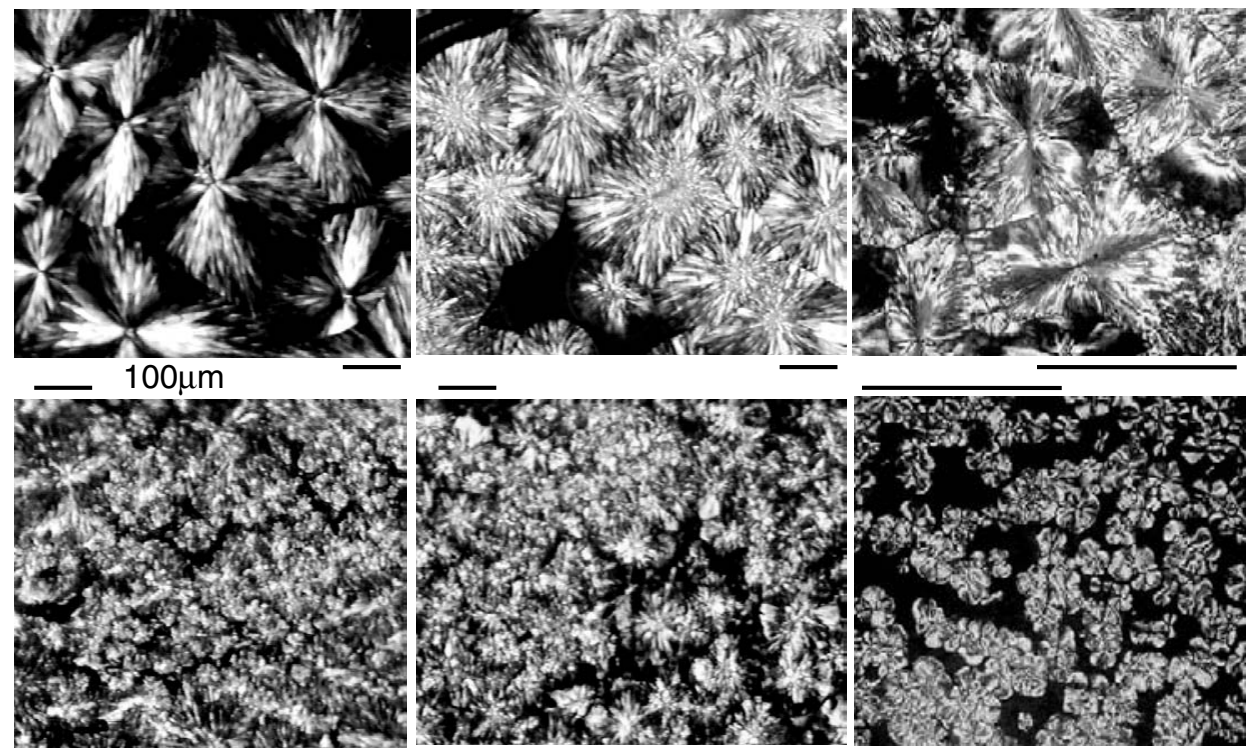

FIGURE 2.6 Comparison of cross-polarized optical microscopy pictures of unfilled polymers (top) and their respective nanocomposites (bottom) with $3 \mathrm{wt} \%$ of montmorillonite fillers; PEO (left), PP-g-MA (middle), and sPS (right).

systems. ${ }^{11,56,64,75-78}$ For example, polypropylene crystallization in the presence of nanotubes shows increased crystallization temperature and rate of crystallization with the introduction of varying concentrations of nanotubes, ${ }^{79,80}$ with no change in the crystalline structure or the melting point. Furthermore, the PP crystallite size decreases in the presence of nanotubes, ${ }^{64,75,76}$ in agreement with the general behavior observed in polymer/layered-inorganic nanocomposites.

When nanotubes interact strongly with the host polymer, as for example with conjugated and ferroelectric polymers, polymer crystallization is altered, developing higher-order structures and increased degrees of crystallinity. ${ }^{79,80}$ However, the crystallization effects discussed above for layered silicates due to polymer coordination with alkali cations (for PEO) and due to extensive hydrogen bonding (for amides) are, as expected, absent in nanotube-reinforced nanocomposites. Namely, PEO does not have any amorphous regions near nanotubes, and it follows bulklike crystallization, with the overall percent crystallinity, crystallization point, and melting point remaining unaffected, even at loadings of 7 wt $\%$ nanotubes. ${ }^{81}$ Similarly, polyamide- 6 and polyamide- 12 matrices reinforced with nanotubes exhibit crystallization similar to that of the unfilled polymer. ${ }^{78,82}$

Finally, the one-dimensional geometry of nanotubes provides exciting opportunities for controlled nucleation and growth of single crystals along individual fibers, as for example with polyamide- 6,6 and polyethylene crystallized from solution, allowing for control of crystallite periodicity and molecular-level architecture. ${ }^{83}$ This unique capability of nanotubes can conceivably lead to special types of "functionalization" of individual nanotubes, which can be exploited to improve interactions (see our discussion of better interfacial coupling) and control dispersion in selected polymer matrices. 


\subsubsection{Effects on Mechanical Properties}

Most polymer-clay nanocomposite studies report tensile properties as a function of MMT content $\left(\phi_{\mathrm{MMT}}\right)$. As a typical example, in Figure 2.7 we compare tensile moduli from various studies of neat PP/o-MMT and MA-functionalized-PP/oMMT nanocomposites. The characteristic behavior for polymer/layered-inorganic nanocomposite materials ${ }^{6,8}$ is observed: Namely, there is a sharp increase in Young's modulus for very small inorganic loadings $\left(\phi_{\mathrm{O}-\mathrm{MMT}}<4 \mathrm{wt} \%\right)$, followed by a much slower increase beyond $\phi_{\mathrm{O}-\mathrm{MMT}} \simeq 5 \mathrm{wt} \%$. With increasing $\phi_{\mathrm{MMT}}$, the yield stress does not change markedly compared to the neat-polymer value, and there is only a small decrease in the maximum strain at break. PP systems filled conventionally (i.e., no nanometer-level dispersion) by the same fillers (e.g., 2C18-MMT) do not exhibit as large increases in their tensile modulus (Figure 2.7a).

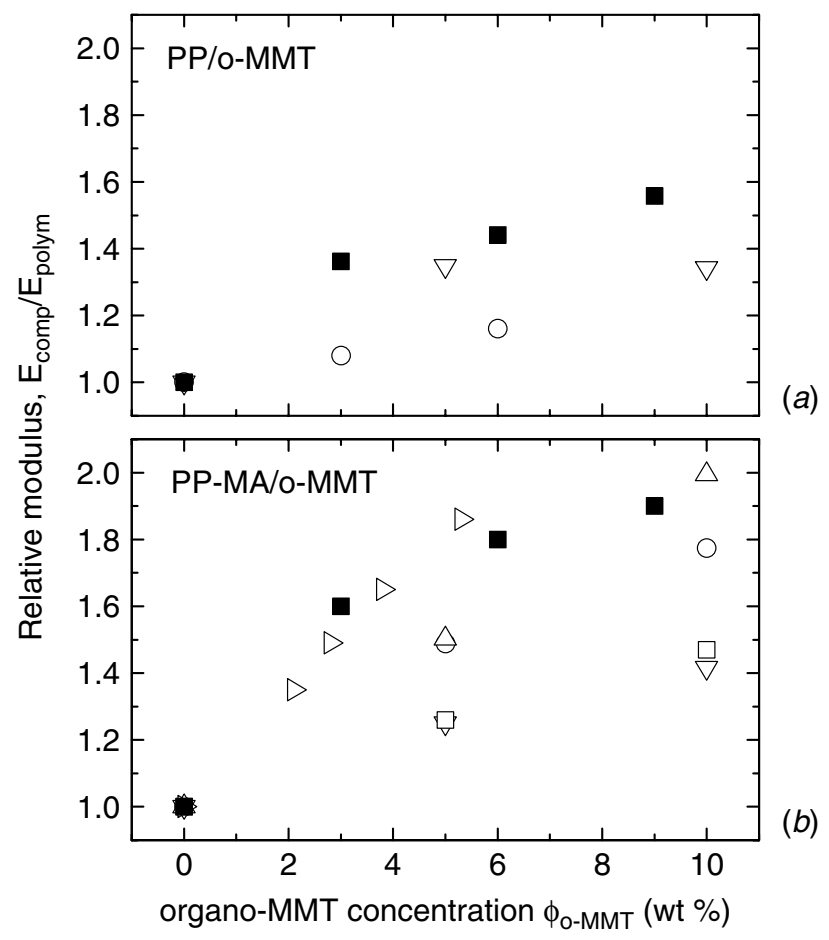

FIGURE 2.7 Tensile moduli (relative to bulk value) for various PP-MMT nanocomposites. (a) neat-PP hybrids: with f-MMT $\left(\square^{49}\right)$, C18-MMT $\left(\nabla^{35}\right)$, and 2C18-MMT $\left(\mathrm{O}^{49}\right)$. In the absence of favorable thermodynamics, the dispersion and thus the mechanical properties are a strong function of the processing conditions. (b) PP-g-MA/2C18-MMT melt-processed nanocomposite $\left(\boldsymbol{\square}^{49}\right)$ and PP hybrids formed via various PP-g-MA pretreated o-MMT master batches: C18-MMT $\left(\triangleright^{34}\right)$ and C18-MMT $\left(O, \triangle^{35}\right)$. Given the well-defined thermodynamics of mixing, there is a small variation of dispersion and mechanical properties across different systems and various research groups. Slight changes in the thermodynamics [e.g. when a different surfactant is employed C8-MMT $\left(\nabla, \square^{35}\right)$ ] result in moduli changes. (Adapted from Ref. 49, copyright (C) 2001, American Chemical Society, with permission.) 
This mechanical reinforcement is expected and not too exciting at first glance, especially considering that the montmorillonite filler platelets have a very high intrinsic stiffness (tensile modulus of 140 to $180 \mathrm{GPa}$ ). However, there are some points that can be made: The tensile results obtained from thermodynamically stable hybrids are not affected by processing conditions (since the nanocomposite structure remains the same), whereas in the absence of favorable PP/o-MMT thermodynamics, the structure and tensile properties vary strongly with the processing conditions (Figure 2.7b). Similar improvements in mechanical properties can also be achieved by other layered particulate fillers; however, much higher filler loadings are required (e.g., by loading 30 to $60 \mathrm{wt} \%$ of talc or mica ${ }^{14}$ ), since such particles are not well dispersed and the effective filler surface area is orders of magnitude smaller. Finally, for PP/o-MMT, the relative improvement in the moduli compared to the unfilled polymer is rather small (barely reaching $60 \%$ for PP and $100 \%$ for PP-g-MA), whereas in other systems, such as elastomers or polyethylene, improvements of 400 to $1200 \%$ in the Young's modulus can be achieved by the same o-MMT fillers. The origin of this behavior is traced to two effects:

1. The relatively poor interaction of polyolefins with o-MMT [cf. eq. (2.6); interfacial adhesion energy of $\sim 83 \mathrm{~mJ} / \mathrm{m}^{2}$; see also Sec. 11.2 in Israelachvili ${ }^{32}$ ]. As the polymer-inorganic adhesion is improved (e.g., when MA functional groups are added to the polymer), the stresses are much more effectively transferred from the polymer matrix to the inorganic filler, and thus a higher increase in Young's modulus is achieved (Figure 2.7b).

2. The relatively high modulus of the original polymer (for the PP reported, 0.6 to $1.3 \mathrm{GPa}$ ). The latter effect becomes clearer when this behavior is contrasted with nanocomposites formed by the same filler in a "softer" matrix, such as elastomers or PEs that have tensile moduli in the range 0.1 to $0.3 \mathrm{GPa}$.

As further evidence of the last two points, we also show the tensile moduli of polyamide-MMT systems (Figure $2.8 a$ ), where substantial improvements in mechanical properties can be achieved, despite the relatively high stiffness of the polymer matrix, due to the very effective stress transfer from the polymer to the filler, mediated by strong hydrogen bonding. In the case of polyamide-6/MMT nanocomposites, independent of the original polyamide- 6 matrix characteristics and of whether the hybrids were formed by in situ polymerization or melt blending, ${ }^{17,18,42-44,84}$ there seems to be considerable agreement on the enhancement achieved in the tensile modulus that spans research groups, methods, and materials. We postulate that due to the strong interfacial adhesion (i.e., every amide group of the polymer can hydrogen bond to the silicate surface), the interfacial strength and maximum interfacial shear stress are dictated by the polymer-MMT interactions and overwhelm all other parameters that relate to processing and dispersion, polymer matrix characteristics, and/or stiffness of the filler. 


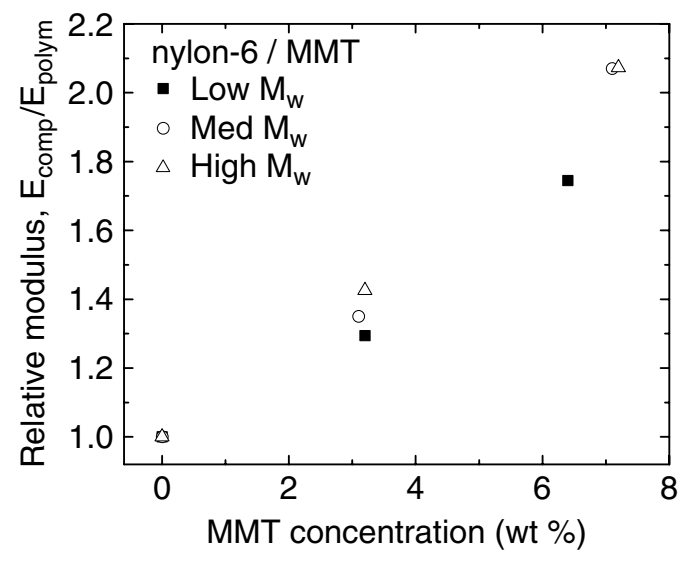

(a)

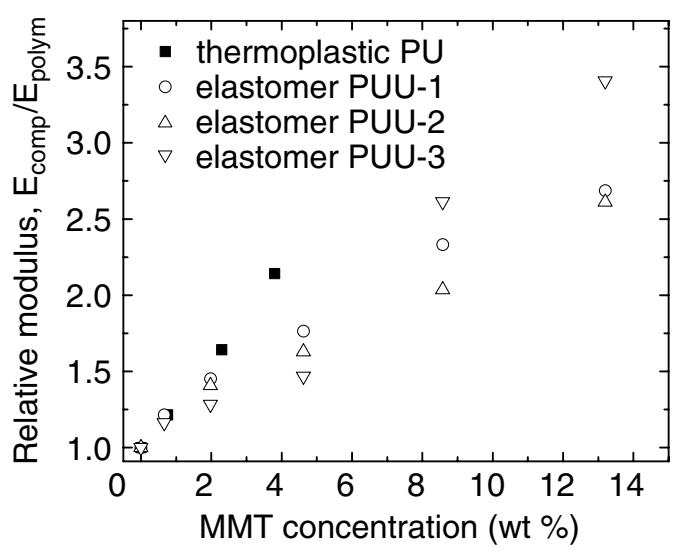

(b)

FIGURE 2.8 Tensile moduli (relative to bulk value) for various nanocomposites: $(a)$ polyamide-6/MMT nanocomposites, ${ }^{84}$ with low-, medium-, and high- molecular weight polyamide- 6 matrix, as an example of high improvement in mechanical properties due to effective stress transfer from polymer to filler; $(b)$ polyurethane and polyurethane copolymers/MMT nanocomposites, as an example of high improvement in mechanical properties due to a "soft" original polymer $\left(\mathbf{\square}^{85}, \nabla^{40}, \bigcirc \Delta^{41}\right.$.)

2.3.2.1 Theoretical Insights in to Mechanical Properties Even from the very brief discussion above, it becomes obvious that a priori prediction of the mechanical properties of polymer-inorganic nanocomposites is rather involved, and to date the design of such nanocomposites is based mostly on Edisonian approaches. Theoretical models developed for the prediction of mechanical properties of conventional composites, such as the Halpin-Tsai ${ }^{86}$ and Mori-Tanaka ${ }^{87}$ models, fail in their "straightforward" application to nanocomposite systems. There are numerous physical phenomena that need to be included in such models so as to better describe the mechanical behavior of polymer-matrix nanocomposite materials. Again drawing examples from polymer/layered-silicate nanocomposites, recent theoretical models have been developed that attempt to capture the behavior of these materials by accounting for the high aspect ratio of the fillers: for example, an effort ${ }^{88}$ that modifies the Halpin-Tsai model to account for the buckling of filler platelets, incomplete dispersion, and nonbiaxial inplane filler orientation; despite its additional complexity and improvements, this modified Halpin-Tsai model does not seem to be highly successful in predicting the mechanical properties of polymer/layered-silicate nanocomposites for a wide range of polymer matrices. ${ }^{88}$ The main shortcoming in the previous approach is attributed 89 to the insufficient modeling of a "constrained region" of polymeric material surrounding the nanoscopic filler; this interfacial polymer is expected to differ in properties and morphology from the bulk polymer matrix, as has been observed experimentally. However, accounting for such a constrained region, as for example by introducing appropriate modifications ${ }^{89,90}$ in the Mori-Tanaka model, still has a limited predictive power when applied 
across various polymer matrices and necessitates adjustment of the model's parameters for each nanocomposite system. ${ }^{90}$ Even in the most focused approach, when a mechanical model is developed to describe a single polymer-inorganic nanocomposite ${ }^{91}$ - while accounting for the imperfect interfacial coupling and the effective aspect ratio and filler volume fraction due to varied dispersion with filler loading - such a model necessitates calculation of an interfacial strength parameter (in this case an interfacial shear stress, which was calculated ${ }^{91}$ to be 2 to $8 \mathrm{MPa}$ for a poly(dimethyl siloxane) (PDMS)/MMT system).

Despite any shortcomings and approximations, these theoretical endeavors offer valuable insights in important design parameters for the mechanical performance of polymer nanocomposites. Specifically:

- Mechanical properties are determined by the effective filler aspect ratio and effective filler volume fraction when incomplete dispersion is accounted for $^{88,91}$ (rather than on the absolute filler loading and the aspect ratio of the individual fillers).

- Filler-specific mechanisms of deformation and fracture can have a considerable contribution to the mechanical properties of the nanocomposite. ${ }^{88}$

- The correct enumeration of the interfacial strength is crucial for correct estimation of the composite's mechanical properties, ${ }^{91,90}$ and its small value compared to the modulus of the filler can dramatically limit a filler's reinforcing effectiveness.

In particular for the last item, interfacial strength at the polymer-filler interface can be experimentally measured directly in very few cases; for example, carbon nanotubes have been pulled out from a polymer [poly(ethylene-butene)] matrix by AFM, yielding interfacial strengths ${ }^{92,93}$ of 10 to $90 \mathrm{MPa}$, depending on the nanotube radius. These experimental interfacial strength values correlate well with interfacial forces calculations, ${ }^{30}$ such as those described earlier [eq. (2.6)]. Thus, one may expect that the same approach used for predicting miscibility of polymers and layered fillers may be helpful in estimating the polymer-filler interfacial strength. Given the continuum character and the assumptions behind such calculations, and the very approximate numbers available for the surface tension components of the materials involved, this approach can only provide a first-order estimation of the interfacial strength for polymer and various nanofillers. Albeit its uncertainty, this theoretical value of the polymer-filler interfacial strength may be an important design element for the mechanical properties of nanocomposites, especially since it is very difficult to envision approaches able to determine this interfacial strength experimentally.

Some examples of the application of eq. (2.6) for polymer/layered-inorganic nanocomposites could be:

- For polypropylene-montmorillonite interfaces, ignoring all necessary functionalizations for PP would yield an interfacial adhesive energy of 
$\sim 83 \mathrm{~mJ} / \mathrm{m}^{2}$, corresponding to an interfacial strength of $\sim 10 \mathrm{MPa}$ (cf. 3 to $7 \mathrm{MPa}$ from tensile measurements ${ }^{49}$ ).

- For PDMS-montmorillonite, the same approach yields an interfacial energy of $\sim 91 \mathrm{~mJ} / \mathrm{m}^{2}$ or an interfacial strength of $\sim 11 \mathrm{MPa}$ (cf. 2 to $8 \mathrm{MPa}$ from theoretical models ${ }^{91}$ ).

- For polyamide-montmorillonite nanocomposites, and ignoring all crystalline-phase changes that may be caused by silicate fillers, ${ }^{42-44}$ an adhesive interfacial adhesion of $\sim 107 \mathrm{~mJ} / \mathrm{m}^{2}$, corresponding to an interfacial strength of $\sim 14 \mathrm{MPa}$.

- For carbon nanotube-polypropylene yields an interfacial energy of $\sim 49 \mathrm{~mJ} / \mathrm{m}^{2}$ (cf. $47 \mathrm{~mJ} / \mathrm{m}^{2}$ from AFM experiments ${ }^{92}$ ) or an interfacial strength* of $\sim 6.2 \mathrm{MPa}$ (cf. 20 to $40 \mathrm{MPa}$ from multiwalled nanotubes, ${ }^{93}$ and $2 \mathrm{MPa}$ from computer simulations ${ }^{94}$ ).

To the extent that they are valid, the observations above bear significant implications for the possibilities of mechanical property improvements via nanocomposite formation. Specifically:

1. Given the nature of a polymer (i.e., $\gamma^{\mathrm{LW}}$ and $\gamma^{ \pm}$), the maximum mechanical reinforcement by a completely dispersed nanofiller will be limited by the polymer-filler interfacial strength. For example, in the case of PE and PP $\left(\gamma^{\mathrm{LW}} \simeq 26 \mathrm{~mJ} / \mathrm{m}^{2}\right.$ and $\left.\gamma^{ \pm}=0\right)$ and layered silicates, there would be a common limit of about 2 to $4 \mathrm{GPa}$ for the maximum tensile modulus that can be achieved through nanocomposite formation. This is in agreement with experimental studies for these systems, which show a similar absolute value for the maximum tensile modulus obtained by PE and PP [albeit reflected in much bigger relative improvements of 400 to $1200 \%$ for the softer LDPE, compared to 60 to $100 \%$ for the stiffer $i$-PP (Figure 2.7)].

2. The addition of a small number of functional groups (e.g. addition of maleic anhydride groups in PP) would increase the interfacial adhesion only moderately, and would similarly increase the tensile moduli only moderately (Figure 2.7b).

3. The addition of large numbers of strongly interacting (with the filler) groups along the chain, such as hydrogen-bonding groups densely across the polymer backbone, would result in larger relative improvements in mechanical properties (Figure 2.8), but still below the upper limits set by the interfacial adhesions calculated. (The use of polyamide- 6 as an example in this case is questionable, given the promotion of the $\gamma$-phase crystal for the

\footnotetext{
*Equation (2.6) is independent of geometry; however when estimating interfacial strength, the filler geometry (i.e., contact geometry) must be considered (see, e.g., Sec. 11.1 in Israelachvili ${ }^{32}$ or Sec. VI.1 in Van $\left.\mathrm{Oss}^{29}\right)$. The value provided for the nanotube-polyethylene here $(6.2 \mathrm{MPa})$ is based on the interaction of two semi-infinite flat surfaces. Calculation for a cylinder in contact with a semi-infinite flat surface yields an interfacial strength of 4.6 $\mathrm{MPa}$, whereas the interaction between a cylinder emerged in a polymer should be somewhere between these two values.
} 
nanocomposites ${ }^{42-44}$; however, the favorable comparison of polyamide-6 behavior with the behavior of urethane-urea systems may a posteriori justify this choice.)

4. Finally, although chemical bonding of the polymer to the filler may seem the ultimate route to reinforce the polymer-filler interface, if such covalent bonds are not introduced densely across the length of the polymer, they will result in only a limited interfacial reinforcement and a respectively moderate improvement in the mechanical properties. This has been shown in crosslinked systems with reactive (via the cross-linking groups) dispersion of silicate layered fillers. ${ }^{95}$

\subsubsection{Effects on Barrier Properties}

The permeability of small penetrant molecules through an organic matrix is determined by the solubility and diffusivity of the small molecule in the matrix as well as by the mean-square displacement (total path length traveled) divided by the sample thickness. In principle, the addition of a filler in the polymer matrix is expected to affect the solubility and diffusivity of a penetrant molecule, especially in the vicinity of the filler (i.e., in the filler-polymer interfacial region and at least one polymer $R_{g}$ away from the filler surface). Also, it is expected that fillers will affect the path tortuosity (mean-square displacement of penetrant versus film thickness) directly, when penetrants are forced to travel around impermeable fillers, and indirectly, when fillers induce polymer chain alignment or alignment and modification of polymer crystallites.*

Theoretical approaches on the barrier properties of nanocomposites treat fillers as impermeable nonoverlapping particles and assume no permeability changes in the polymer matrix. ${ }^{97-100}$ Effectively, this means that the permeability of the composite will be smaller than the permeability of the matrix (unfilled polymer) by a factor equal to path tortuosity in the composite (simply assuming that the penetrant path cannot cross any filler particles). This path tortuosity was calculated by Nielsen ${ }^{97}$ for completely aligned filler particles (all fillers have their larger surface parallel to the film surfaces, but there is no order in the filler center of mass), and its contribution to the composite permeability was derived to be

$$
\frac{P_{\text {comp }}}{P_{\text {poly }}}=\frac{1-\phi}{1+a \phi}
$$

with $a$ being the filler aspect ratio (for square fillers of length/width $L$ and thickness $\mathrm{W}, a=\mathrm{L} / 2 \mathrm{~W}$ ) and $\phi$ the volume fraction of the filler. Bharadwaj ${ }^{100}$

*The first mechanism, associated with chain alignment and the related diffusive anisotropy of a small molecule within aligned chains, has a relatively weak effect on permeability, ${ }^{96}$ whereas the second mechanism, associated with crystallite alignment and changes in the crystal morphologies, causes rather strong changes in permeability and is commonly employed in strain-hardened semicrystalline polymers for barrier applications. 

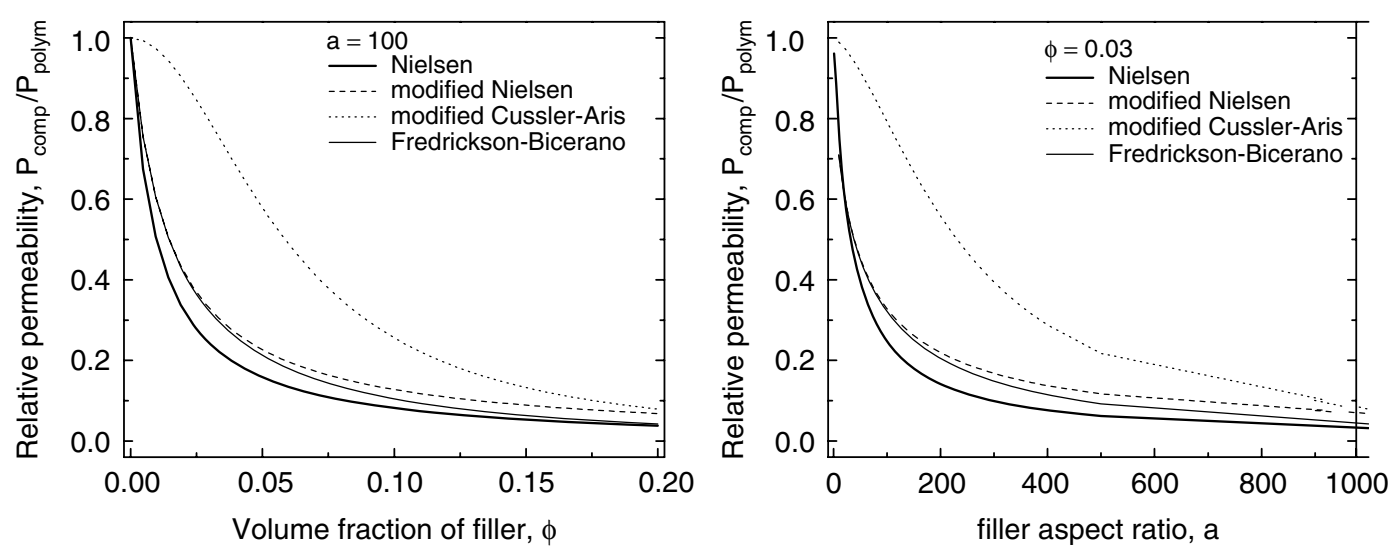

FIGURE 2.9 Comparison of theoretical models quantifying the effect of path tortuosity on the permeability of a composite: Nielsen model ${ }^{97}$ [eq. (2.8)], Friedrickson-Bicerano [eq. (2.10)], modified Nielsen [eq. (2.9)], and Cussler-Aris [eq. (2.11)].

has modified this equation to account for nonaligned fillers by introducing an order parameter $S$ for the filler orientation:

$$
\frac{P_{\text {comp }}}{P_{\text {poly }}}=\frac{1-\phi}{1+a \phi \frac{2}{3}\left(S+\frac{1}{2}\right)} \quad \text { with } \quad S=\frac{1}{2}\left\langle 3 \cos ^{2} \theta-1\right\rangle=\left\{\begin{array}{cc}
1 & \| \text { surface } \\
0 & \text { random } \\
-\frac{1}{2} & \perp \text { surface }
\end{array}\right.
$$

which reduces to Nielsen's equation for perfectly aligned fillers $(S=1)$. In a more detailed approach, Friedrickson and Bicerano ${ }^{99}$ derived the same path tortuosity effects for circular fillers (radius $R$ and thickness $2 W$ ) and an aspect ratio $a=R / 2 W$ :

$$
\frac{P_{\text {comp }}}{P_{\text {poly }}}=\frac{1}{4}\left(\frac{1}{1+a \phi \beta_{1}}+\frac{1}{1+a \phi \beta_{2}}\right)^{2} \quad \text { with }\left\{\begin{array}{l}
\beta_{1}=(\pi / \ln a)(2-\sqrt{2}) / 4 \\
\beta_{2}=(\pi / \ln a)(2+\sqrt{2}) / 4
\end{array}\right.
$$

which can cover a wider $\phi$ range, from dilute to semidilute, than the modified Nielsen and modified Cussler-Aris relations (as presented in the same work, ${ }^{99}$ modified to address circular fillers):

$$
\frac{P_{\text {comp }}}{P_{\text {poly }}}=\frac{1}{1+a \phi \pi / \ln a}\left(\begin{array}{c}
\text { modified } \\
\text { Nielsen }
\end{array}\right), \quad \frac{1}{1+[a \phi \pi /(4 \ln a)]^{2}}\left(\begin{array}{c}
\text { modified } \\
\text { Cussler-Aris }
\end{array}\right)
$$

Nevertheless, eq. (2.10) generally gives results similar to those using the Nielsen approach [eq. (2.8)], when a geometric correction of $\sqrt{\pi} / 2$ is applied to the filler aspect ratio (i.e., comparing equal area fillers, square for Nielsen and circular for Friedrickson-Bicerano). A comparison of the theoretical models is illustrated in Figure 2.9. Given that all models (except the Cussler-Aris) give similar behavior for the range of parameters relevant to polymer/layered-inorganic nanocomposites $(10<a<1000$ and $\phi \leq 15 \mathrm{vol} \%)$, we henceforth use the much 
simpler Nielsen model, including the addition of the orientation term [eqs. (2.8) and (2.9)]. According to this model, the obvious expectations can be quantified: higher aspect ratio fillers provide substantial lower permeabilities for a given filler volume fraction (Figure 2.10a), and aligned fillers are more effective barriers for a given aspect ratio and filler loading (Figure 2.10c). Additionally, some not-so-obvious conclusions can also be drawn:

- Beyond the filler aspect ratio, the composite permeability is also controlled by the filler volume fraction and/or by filler alignment [e.g., eq. (2.9)]: Thus, low aspect ratio fillers can be as effective as higher aspect ratio fillers, although at slightly higher loadings. For example, for aligned fillers (Figure 2.10a), a completely exfoliated montmorillonite $(a=500)$ at $\phi \simeq$ $2 \mathrm{vol} \%$ has comparable permeability with a partially exfoliated montmorillonite $(a=200)$ at $\phi \simeq 3 \%$, or a mostly intercalated montmorillonite $(a=$ $100)$ at $\phi \simeq 5 \%$. This observation has important implications in designing a barrier nanocomposite: For the same example, instead of completely exfoliating a given filler, a task that is usually difficult to achieve, the same filler in a partially exfoliated or mostly intercalated morphology could achieve the same barrier performance at slightly higher filler loadings.

- Perfectly aligned fillers result in similar permeabilities with randomly oriented fillers of higher aspect ratio and/or at higher loading. For example (Figure 2.10b), for an $a=300$ filler, perfect alignment at $\phi=1.5 \%$ results in the same barrier performance as that of the same filler when randomly oriented at $\phi \simeq 4.3 \%$; and for an $a=500$ filler, perfect alignment at 1.5 vol\% is comparable in permeability with a $4.5 \mathrm{vol} \%$ composite with random filler orientation. Along the same lines, a perfectly aligned filler nanocomposite with $a=300$ at $\phi=1 \%$ has similar barrier performance as $a=500$ at $2 \%$. This observation also provides important guidance on how to avoid the difficult task of perfectly aligning the fillers parallel to the film surface (Figure 2.10c).

- The effect of filler orientation on permeability decreases in importance with a higher filler aspect ratio (Figure 2.10c). For example, permeability improvement for $a=1000$ is only 5\% better with perfect alignment $(S=1)$ than with a random orientation $(S=0)$, and for $a=500$ this difference is about $10 \%$.

Additionally, the favorable comparison of these theoretical predictions with experimental data (Figure $2.10 d$ ) gives some credibility to the conclusions above. In Figure $2.10 d$ we plot experimental water vapor permeabilities of various solvent cast nanocomposite films. The experimental behavior follows closely the theoretical trend and is enclosed between the response of exfoliated systems (especially for low filler loadings) and that of intercalated systems (for moderate and higher loadings). This reflects the same effective filler aspect ratio 


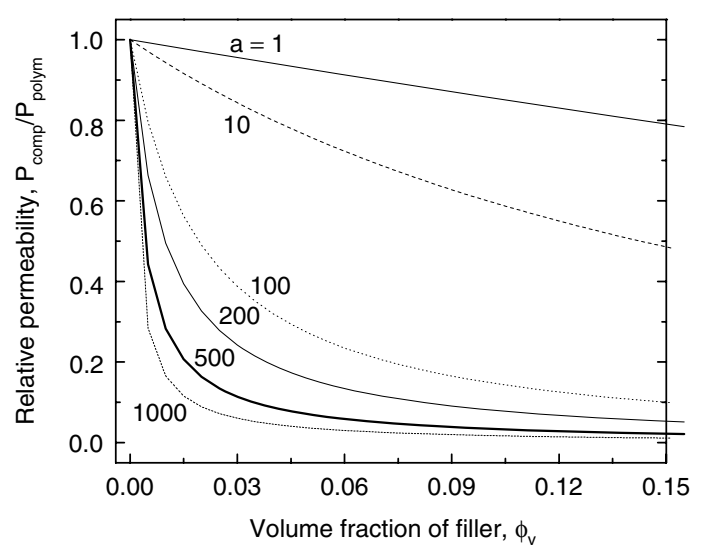

(a)

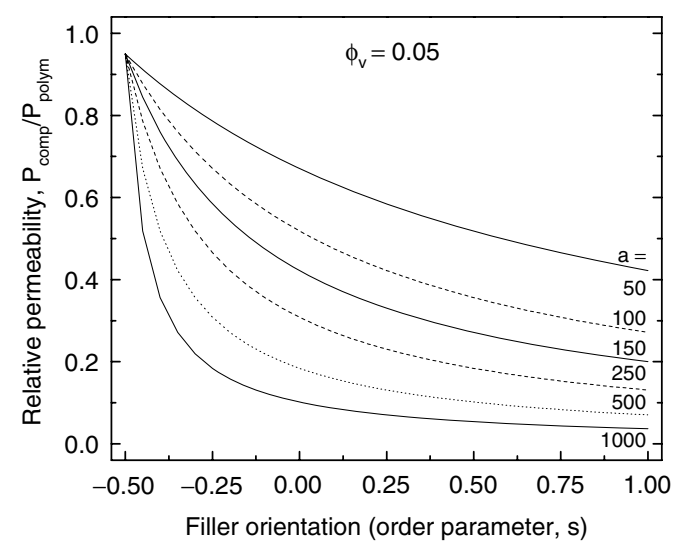

(c)

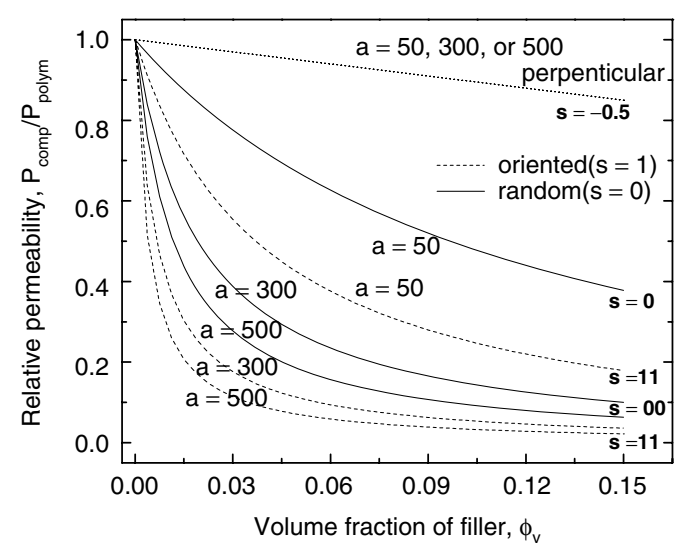

(b)

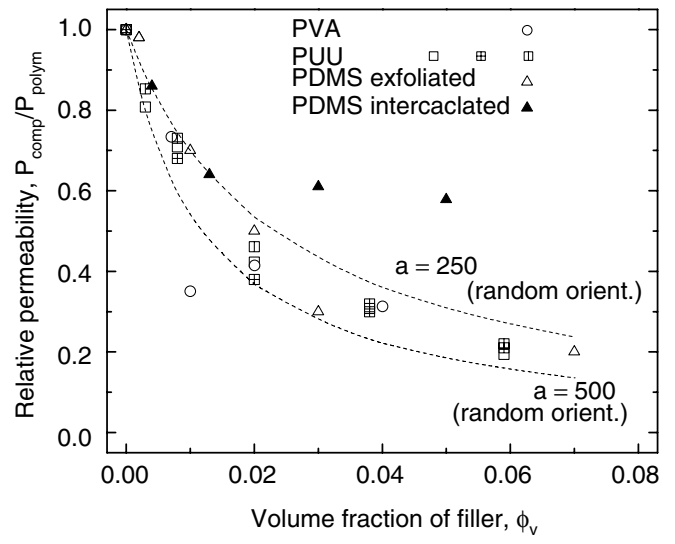

(d)

FIGURE 2.10 Theoretical predictions based on path tortuosity [eq. (2.9)], as a function of (a) filler aspect ratio $a=1$ to 1000 ; $(b)$ filler aspect ratio and alignment $(S=1$ : perfect smectic alignment-dashed lines; $S=0$ : random orientation — solid lines); (c) filler aspect ratio for a constant volume fraction $\phi_{V}=5 \%$. (d) Comparison of the same theoretical predictions (parameters as indicated) with experimental values for water vapor permeabilities in various polymer-montmorillonite nanocomposites. (From Refs. 39-41.)

discussed before in relation to mechanical properties. This agreement persists for all systems that have good filler dispersion (as achieved by solvent casting) and disappears for the same polymer and filler when dispersion is poor (cf. PDMS/dimethyldialkyl-montmorillonite with intercalated versus exfoliated composite structures). Finally, this agreement is rather independent of polymer and filler hydrophillicity, ranging from very hydrophillic poly(vinyl alcohol) reinforced by $\mathrm{Na}^{+}$-montmorillonite, to moderate poly(urethane-co-ureas) to rather hydrophobic poly(dimethyl siloxane) and dialkyl-modified montmorillonite. This agreement is also independent of polymer crystallinity: ranging from semicrystalline poly(vinyl alcohol) with filler-induced crystallinity effects, to segmented semicrystalline poly(urethane-co-ureas), to amorphous poly(dimethyl siloxane). Thus, it seems that the path tortuosity effects may in fact overwhelm 
other important parameters (such as permeant solubility changes* and polymer crystallinity effects) when it comes to predicting permeability changes upon nanocomposite formation.

\subsection{FUTURE OUTLOOK}

Nanocomposites, in the sense of hybrid materials with novel properties beyond the realm of unfilled polymers and conventional composites, bear high promise for enabling new uses and applications of polymer materials. In the simplest approach, they can expand the window of applications of a given polymer, and in the best case they can enable the use of polymer-matrix composites in applications where metal or ceramic materials are currently used. One of the first untapped challenges in the field is to go beyond the simple dispersion of fillers and move toward the development of methods to create well-defined three-dimensional morphologies of nanofillers: morphologies that contain highly aligned fillers, house-of-cards structures, edge-connected (starlike) formations, and alternating two- and one-dimensional fillers.

The highest benefit of the hybrid character of nanocomposites comes from overcoming the property trade-offs associated with conventional composites: For example, nanocomposites can improve stiffness without sacrificing toughness, can enhance barrier properties without sacrificing transparency, can bestow flame retardancy without sacrificing mechanical properties, and can enhance mechanical performance and biodegradability simultaneously. When such behaviors are enhanced synergistically with effects from other additives or fillers, they can effectively push the envelope of the current state of the art. Such approaches will develop particularly exciting systems where synergistic combinations of multiple nano- and macrofillers are properly combined in a multifiller composite material.

Although it currently engages an overwhelming number of research groups, the field desperately needs well-designed scientifically-based studies to explore the fundamentals of these materials. Since barriers to entering the field are really low (no need for special equipment or expensive materials; studies can be published even when reproducing results from previous works or making minor incremental advances), the temptation is high to simply mix polymers with off-the-shelf nanofillers and just report x-ray diffraction and mechanical measurements. The real potential of these materials will remain untapped, however, until the nanoscale mechanisms responsible for macroscopic properties are unveiled and are further exploited to make radically new materials. New horizons need to be explored, especially outside the "comfort zone" of traditional polymer or materials scientists. If one considers the numerous examples of biological organic and inorganic nanostructures with unparalleled performances and combinations

*We should point out that barrier or permeability properties relate to the rate of permeant molecule diffusion through a polymer or nanocomposite material and cannot be extended to make predictions for ultimate water uptake or more general solvent uptake. If water or solvent uptake is of interest, an independent experiment is required, and for this property, the changes in solubility upon nanocomposite formation are the determining factor. 
of properties that transcend any synthetic material, one can only start to imagine the limitless possibilities of this field.

\section{Acknowledgments}

Experimental data, theoretical arguments, conclusions, and opinions presented in this chapter have been developed through our research in this field over the last few years. These efforts were supported financially by the Pennsylvania State University, the National Science Foundation, the Office of Naval Research, the ACS/Petroleum Research Fund, the National Research Council, and various industrial projects, including Projects from Sumitomo Chemical, Air Products, Coca-Cola, and Bayer MaterialScience.

\section{REFERENCES}

1. Theng, B.K.G. Formation and Properties of Clay-Polymer Complexes. Elsevier, Amsterdam, The Netherlands, 1979.

2. Theng, B.K.G. Chemistry of Clay-Organic Reactions. Wiley, New York, 1974.

3. Pinnavaia, T.J.; Beall, G.W., Eds. Polymer-Clay Nanocomposites. Wiley, Chichester, West Sussex, England, 2000.

4. Utracki, L.A. Clay-Containing Polymeric Nanocomposites. Rapra Technology, Shawbury, Shrewsbury, England, 2004.

5. Mai, Y.; Yu, Z., Eds. Polymer Nanocomposites. Woodhead Publishing, Cambridge, England, 2006.

6. Alexandre, M.; Dubois, P. Polymer-layered silicate nanocomposites: preparation, properties and uses of a new class of materials. Mater Sci. Eng. R Rep. 2000, 28, $1-63$.

7. Giannelis, E.P.; Krishnamoorti, R.K.; Manias, E. Polymer-silicate nanocomposites: model systems for confined polymers and polymer brushes. Adv. Polym. Sci., 1998, 138, 107-148.

8. Ray, S.S.; Okamoto, M. Polymer/layered silicate nanocomposites: a review from preparation to processing. Prog. Polym. Sci. 2003, 28, 1539-1641.

9. LeBaron, P.C.; Wang, Z.; Pinnavaia, T.J. Polymer-layered silicate nanocomposites: an overview. Appl. Clay Sci. 1999, 15, 11-29.

10. Leroux, F.; Besse, J.P. Polymer interleaved layered double hydroxide: a new emerging class of nanocomposites. Chem. Mater. 2001, 13, 3507-3515.

11. Thostenson, E.T.; Ren, Z.F.; Chou, T.W. Advances in the science and technology of carbon nanotubes and their composites: a review. Compos. Sci. Technol. 2001, 61, 1899-1912.

12. Solomon, D.H.; Hawthorne, D.G. Chemistry of Pigments and Fillers. R.E. Krieger, Malabar, FL, 1991.

13. Al-Malaika, S.; Golovoy, A.; Wilkie, C.A., Eds. Chemistry and Technology of Polymer Additives. Blackwell Science, Oxford, England, 1999.

14. Karian, H.G., Ed. Handbook of Polypropylene and Polypropylene Composites. Marcel Dekker, New York, 1999.

15. Lu, C.; Mai, Y.-M. Influence of aspect ratio on barrier properties of polymer-clay nanocomposites. Phys. Rev. Lett. 2005, 95, 088303. 
16. Mitchell, C.A.; Bahr, J.L.; Arepalli, S.; Tour, J.M.; Krishnamoorti, R. Dispersion of functionalized carbon nanotubes in polystyrene. Macromolecules 2002, 35, 88258830 .

17. Kojima, Y.; Usuki, A.; Kawasumi, M.; Okada, A.; Fukushima, Y.; Kurauchi, T.T.; Kamigaito, O. Synthesis and mechanical properties of nylon-6/clay hybrid. J. Mater Res. 1993, 8, 1179-1184, 1185-1189.

18. Kojima, Y.; Usuki, A.; Kawasumi, M.; Okada, A.; Kurauchi, T.T.; Kamigaito, O. Synthesis of nylon-6/clay hybrid by montmorillonite intercalated with $\epsilon$-caprolactam. J. Polym. Sci. A Polym. Chem. 1993, 31, 983-986.

19. Vaia R.A.; Ishii, H.; Giannelis, E.P. Synthesis and properties of 2-dimensional nanostructures by direct intercalation of polymer melts in layered silicates. Chem. Mater. 1993, 5, 1694-1696.

20. Lan, T.; Kaviratna, P.D.; Pinnavaia, T.J. Mechanism of clay tactoid exfoliation in epoxy-clay nanocomposites. Chem. Mater. 1995, 7, 2144-2150. Wang, M.S.; Pinnavaia, T.J. Clay polymer nanocomposites formed from acidic derivatives of montmorillonite and an epoxy-resin. Chem. Mater. 1994, 6, 468-474. Pinnavaia, T.J. Intercalated clay catalysts. Science 1983, 220, 365-371.

21. Giannelis, E.P.; et al. Structure and dynamics of polymer/layered silicates nanocomposites. Chem. Mater. 1996, 8, 1728-1764. Polymer layered silicate nanocomposites. Adv. Mater. 1996, 8, 29-35. Nanostructure and properties of polysiloxane-layered silicate nanocomposites. J. Polym. Sci. B Polym. Phys. 2000, 38, 1595-1604.

22. Vaia, R.A.; Jandt, K.D.; Kramer, E.J.; Giannelis, E.P. Microstructural evolution of melt intercalated polymer-organically modified layered silicates nanocomposites. Chem. Mater. 1996, 8, 2628-2635. Vaia, R.A.; Price, G.; Ruth, P.N.; Nguyen, H.T.; Lichtenhan, J. Polymer/layered silicate nanocomposites as high performance ablative materials. Appl. Clay Sci. 1999, 15, 67-92.

23. Kanatzidis, M.G.; Wu, C.-G.; Marcy, H.O.; DeGroot, D.C.; Kannewurf, C.R. Conductive polymer-oxide bronze nanocomposites: intercalated polythiophene in $\mathrm{V}_{2} \mathrm{O}_{5}$ xerogels. Chem. Mater. 1990, 2, 222-224. Intercalation of poly(ethylene oxide) in vanadium pentoxide xerogel. Chem. Mater. 1991, 3, 992-994. Synthesis, structure, and reactions of poly(ethylene oxide)/ $\mathrm{V}_{2} \mathrm{O}_{5}$ intercalative nanocomposites. Chem. Mater. 1996, 8, 525-535.

24. Iijima, S. Helical microtubules of graphitic carbon. Nature 1991, 354, 56-58.

25. Vaia, R.A.; Giannelis, E.P. Lattice model of polymer melt intercalation in organically-modified layered silicates. Macromolecules 1997, 30, 7990-7999.

26. Vaia, R.A.; Giannelis, E.P. Polymer melt intercalation in organically-modified layered silicates: Model predictions and experiment. Macromolecules 1997, 30, 80008009. Equation (2.2) in this chapter differs from equation (6) in Ref. 26. Private discussions with R.A. Vaia clarified that Equation (2.2) as provided here is correct, and is the same as the authors used for their calculations in Refs. 26 and 25. Equation (6) in Ref. 26 was wrongly put in press due to a typographical error.

27. Balazs, A.C.; Singh, C.; Zhulina, E. Modeling the interactions between polymers and clay surfaces through self-consistent field theory. Macromolecules 1998, 31, 8370-8381.

28. van Oss, C.J.; Chaudhury, M.K.; Good, R.J. Interfacial Lifschitz-van der Waals and polar interactions in macroscopic systems. Chem. Rev. 1988, 88, 927-941. 
29. van Oss, C.J.; Interfacial Forces in Aqueous Media. Marcel Dekker, New York, 1994.

30. Nuriel, S.; Liu, L.; Barber, A.H.; Wagner, H.D. Direct measurement of multiwall nanotube surface tension. Chem. Phys. Lett. 2005, 404, 263-266.

31. Wu, W.; Giese, R.F.; van Oss, C.J.; Evaluation of the Lifshitz-van der Waals/acidbase approach to determine surface tension components. Langmuir, 1995, 11, 379382.

32. Israelachvili, J. Intermolecular and Surface Forces. Academic Press, San Diego, CA, 1991.

33. Wang, Z.-M.; Nakajima, H.; Manias, E.; Chung, T.C. Exfoliated PP/clay nanocomposites using ammonium-terminated $\mathrm{PP}$ as the organic modification for montmorillonite. Macromolecules 2003, 36, 8919-8922.

34. Hasegawa, N.; Kawasumi, M.; Kato, M.; Usuki, A.; Okada, A. Preparation and mechanical properties of polypropylene-clay hybrids using a maleic anhydridemodified polypropylene oligomer. J. Appl. Polym. Sci. 1998, 67, 37-92.

35. Reichert, P.; Nitz, H.; Klinke, S.; Brandsch, R.; Thomann, R.; Mülhaupt, R. Polypropylene/organoclay nanocomposite formation: influence of compatibilizer functionality and organoclay modification. Macromol. Mater. Eng. 2000, 275, 8-17.

36. Kato, M.; Usuki, A.; Okada, A. Synthesis of polypropylene oligomer-clay intercalation compounds. J. Appl. Polym. Sci. 1997, 66, 1781-1785.

37. Kawasumi, M.; Hasegawa, N.; Kato, M.; Usuki, A.; Okada, A. Preparation and mechanical properties of polypropylene-clay hybrids. Macromolecules 1997, 30, 6333-6338.

38. Wang, K.; Liang, S.; Du, R.N.; Zhang, Q.; Fu, Q. The interplay of thermodynamics and shear on the dispersion of polymer nanocomposite. Polymer 2004, 45, $7953-7960$.

39. Strawhecker, K.; Manias, E. Structure and properties of poly(vinyl alcohol)/ $/ \mathrm{Na}^{+}$ montmorillonite hybrids. Chem. Mater. 2000, 12, 2943-2949.

40. Xu, R.; Manias, E.; Snyder, A.J.; Runt, J. New biomedical poly(urethane urea)layered silicate nanocomposites. Macromolecules 2001, 34, 337-339.

41. Xu, R.; Manias, E.; Snyder, A.J.; Runt, J. Low permeability polyurethane nanocomposites. J. Biomed. Mater. Res. 2003, 64A, 114-119.

42. Lincoln, D.M.; Vaia, R.A.; Wang, Z.G.; Hsiao, B.S.; Krishnamoorti, R. Temperature dependence of polymer crystalline morphology in nylon 6/montmorillonite nanocomposites. Polymer 2001, 42, 9975-9985.

43. Lincoln, D.M.; Vaia, R.A.; Wang, Z.G.; Hsiao, B.S. Secondary structure and elevated temperature crystallite morphology of nylon-6/layered silicate nanocomposites. Polymer 2001, 42, 1621-1631.

44. Lincoln, D.M.; Vaia, R.A.; Krishnamoorti, R. Isothermal crystallization of nylon6/montmorillonite nanocomposites. Macromolecules 2004, 37, 4554-4561.

45. Morgan, A.B.; Harris, J.D. Exfoliated polystyrene-clay nanocomposites synthesized by solvent blending with sonication. Polymer 2004, 45, 8695-3703.

46. Wolf, D.; Fuchs, A.; Wagenknecht, U.; Kretzschmar, B.; Jehnichen, D.; Häussler, L. Nanocomposites of polyolefin clay hybrids, in: Proceedings of Eurofiller'99, LyonVilleurbanne, France, 1999, pp. 6-9. 
47. Vaia, R.A.; Jandt, K.D.; Kramer, E.J.; Giannelis, E.P. Kinetics of polymer melt intercalation. Macromolecules 1995, 28, 8080-8085.

48. Manias, E.; Chen, H.; Krishnamoorti, R.K.; Genzer, J.; Kramer, E.J.; Giannelis, E.P. Intercalation kinetics of poly(styrene)/silicate nanocomposites. Macromolecules 2000, 33, 7955-7966.

49. Manias, E.; Touny, A.; Wu, L.; Strawhecker, K.; Lu, B.; Chung, T.C. Polypropylene/montmorillonite nanocomposite materials: a review of synthetic routes and materials properties. Chem. Mater. 2001, 13, 3516-3523.

50. Oya, A.; Kurokawa, Y.; Yasuda, H. Factors controlling mechanical properties of clay mineral/polypropylene nanocomposites. J. Mater. Sci. 2000, 35, 1045-1050.

51. Messersmith, P.B.; Giannelis, E.P. Polymer-layered silicate nanocomposites: in situ intercalative polymerization of $\epsilon$-caprolactone in layered silicates. Chem. Mater. 1993, 5, 1064-1066.

52. Messersmith, P.B.; Gianellis, E. Synthesis and barrier properties of poly( $\epsilon$-caprolactone)-layered silicate nanocomposites. J. Polym. Sci. Polym. Chem. 1995, 33, $1047-$ 1057.

53. Bahr, J.L.; Tour, J.M. Covalent chemistry of single-wall carbon nanotubes. J. Mater. Chem. 2002, 12, 1952-1958.

54. Sinnott, S.B. Chemical functionalization of carbon nanotubes. J. Nanosci. Nanotechnol. 2002, 2, 113-123.

55. Ramanathan, T.; Liu, H.; Brinson, L.C. Functionalized SWNT/polymer nanocomposites for dramatic property enhancement. J Polym. Sci. B Polym. Phys. 2005, 43; 2269-2279.

56. Xie, X.L.; Mai, Y.-W.; Zhou, X.P. Dispersion and alignment of carbon nanotubes in polymer matrix: a review. Mater. Sci. Eng. R Rep. 2005, 49, 89-112.

57. Wise, K.E.; Park, C.; Siochi, E.J.; Harrison, J.S. Stable dispersion of single wall carbon nanotubes in polyimide: the role of noncovalent interactions. Chem. Phys. Lett. 2004, 391, 207-211.

58. Moon, S.; Jin, F.; Lee, C.-J.; Tsutsumi, S.; Hyon, S.-H. Novel carbon nanotube/ poly( $l$-lactic acid) nanocomposites; their modulus, thermal stability, and electrical conductivity. Macromol. Symp. 2005, 224, 237-295.

59. Paiva, M.C.; Zhou, B.; Fernando, K.A.S.; Lin, Y.; Kennedy, J.M.; Sun, Y-P. Mechanical and morphological characterization of polymer-carbon nanocomposites from functionalized carbon nanotubes. Carbon, 2004, 42, 2849-2854.

60. Putz, K.W.; Mitchell, C.A.; Krishnamoorti, R.; Green, P.F. Elastic modulus of singlewalled carbon nanotube/poly(methyl methacrylate) nanocomposites. J. Polym. Sci. B Polym. Phys. 2004, 42, 2286-2293.

61. Jia, Z.; Wang, Z.; Xu, C.; Liang, J.; Wei, B.; Wu, D.; and Zhu, S. Study on poly(methyl methacrylate)/carbon nanotube composites. Mater. Sci. Eng. A 1999, 271, 395-400.

62. Costache, M.C.; Wang, D.; Heidecker, M.J.; Manias, E.; Wilkie, C.A. The thermal degradation of poly(methyl methacrylate) nanocomposites. Polym. Adv. Technol. 2006, In press.

63. Tang, W.; Santare, M.H.; Advani, S.G. Melt processing and mechanical property characterization of multi-walled carbon nanotube/high density polyethylene (MWNT/HDPE) composite films. Carbon 2003, 41; 2779-2785. 
64. Manchado, M.A.L.; Valentini, L.; Biagiotti, J.; Kenny, J.M. Thermal and mechanical properties of single-walled carbon nanotubes: polypropylene composites prepared by melt processing. Carbon 2005, 43, 1499-1505.

65. Meincke, O.; Kaempfer, D.; Weickmann, H.; Friedrich, C.; Vathauer, M.; Warth, H. Mechanical properties and electrical conductivity of carbon-nanotube filled polyamide-6 and its blends with acrylonitrile/butadiene/styrene. Polymer 2004, 45, 739748.

66. Vaia, R.A.; Liu, W.D. X-ray powder diffraction of polymer/layered silicate nanocomposites: model and practice. J. Polym. Sci. B Polym. Phys. 2002, 40, 1590-1600.

67. Hanley, H.J.M.; Muzny, C.D.; Ho, D.L.; Glinka, C.J.; Manias, E. A SANS study of organo-clay dispersions. Int. J. Thermophys. 2001, 22, 1435-1448.

68. Vaia, R.A.; Liu, W.D.; Koerner, H. Analysis of small-angle scattering of suspensions of organically modified montmorillonite: implications to phase behavior of polymer nanocomposites. J. Polym. Sci. B Polym. Phys. 2003, 41, 3214-3236.

69. Drummy, L.F.; Koerner, H.; Farmer, K.; Tan, A.; Farmer, B.L.; Vaia, R.A. Highresolution electron microscopy of montmorillonite and montmorillonite/epoxy nanocomposites. J. Phys. Chem. B. 2005, 109, 17868-17378.

70. Krishnamoorti, R.; Giannelis, E.P. Strain hardening in model polymer brushes under shear. Langmuir 2001, 17, 1448-1452.

71. Strawhecker, K.; Manias, E. AFM studies of poly(vinyl alcohol) crystallization next to an inorganic surface. Macromolecules 2001, 34, 8475-8482.

72. Giannelis, E.P. unpublished data.

73. Wang, Z.M.; Chung, T.C.; Gilman, J.W.; Manias. E. Melt-processable syndiotactic polystyrene/montmorillonite nanocomposites. J. Polym. Sci. B Polym. Phys. 2003, $41,3173-3137$.

74. Strawhecker, K.; Manias, E. Crystallization behavior of poly(ethylene oxide) in the presence of $\mathrm{Na}^{+}$montmorillonite fillers. Chem. Mater. 2003, 15, 844-849.

75. Bhattacharyya, A.R.; Sreekumar, T.V.; Liu, T.; Kumar, S.; Ericson, L.M.; Hauge, R.H.; Smalley, R.E. Crystallization and orientation studies in polypropylene/single wall carbon nanotube composite. Polymer 2003, 44, 2373-2377.

76. Valentini, L.; Biagiotti, J.; Kenny, J.M.; Santucci, S. Morphological characterization of single-walled carbon nanotubes-PP composites. Compos. Sci. Technol. 2003, 63, 1149-1153.

77. Grady, B.P.; Pompeo, F.; Shambaugh, R.L.; Resasco, D.E. Nucleation of polypropylene crystallization by single-walled carbon nanotubes. J. Phys. Chem. B 2002, 106, $5852-5858$.

78. Sandler, J.K.W.; Pegel, S.; Cadek, M.; Gojny, F.; Es, M.V.; Lohmar, J.; Blau; W.J. Schulte, K.; Windle, A.H.; Shaffer, M.S.P. A comparative study of melt spun polyamide-12 fibres reinforced with carbon nanotubes and nanofibres. Polymer 2004, 45, 2001-2015.

79. Ryan, K.P.; Lipson, S.M.; Drury, A.; Cadek, M.; Ruether, M.; O’Flaherty, S.M.; Barron, V.; McCarthy, B.; Byrne, H.J.; Blau, W.J.; Coleman, J.N. Carbon-nanotube nucleated crystallinity in a conjugated polymer based composite. Chem. Phys. Lett. 2004, 391, 329-333.

80. Zhang, S.; Zhang, N.; Huang, C.; Ren, K.; Zhang, Q. Microstructure and electromechanical properties of carbon nanotube/poly(vinylidene fluoride-trifluoroethylenechlorofluoroethylene) composites. Adv. Mater. 2005, 17, 1897-1901. 
81. Goh, H.W.; Goh, S.H.; Xu, G.Q.; Pramoda, K.P.; Zhang, W.D. Crystallization and dynamic behavior of double-C60-end-capped poly(ethylene oxide)/multi-walled carbon nanotube composites. Chem. Phys. Lett., 2003, 379, 236-241.

82. Liu, T.; Phang, I.Y.; Shen, L.; Chow, S.Y.; Zhang, W.-D. Morphology and mechanical properties of multiwalled carbon nanotubes reinforced nylon-6 composites. Macromolecules 2004, 37, 7214-7222.

83. Li, C.Y.; Li, L.; Cai, W.; Kodjie, S.L.; Tenneti, K. Nanohybrid shishkebabs: periodically functionalized nanotubes. Adv. Mater. 2005, 17, 1198-1202.

84. Fornes, T.D.; Yoon, P.J.; Keskkula, H.; Paul, D.R. Nylon 6 nanocomposites: the effect of matrix molecular weight. Polymer 2001, 42, 9929-9940.

85. Pattanayak, A.; Jana, S.C. Properties of bulk-polymerized thermoplastic polyurethane nanocomposites. Polymer 2005, 46, 3394-3406.

86. Halpin, J.C.; Tsai, S.W. Environmental factors estimation in composite materials design. AFML Trans. 1967, pp. 67-423. Halpin, J.C. Primer on Composite Materials Analysis, 2nd ed. Technomic Publishing, Lancaster, PA, 1992.

87. Mori, T.; Tanaka, K. Average stress in matrix and average energy of materials with misfitting inclusions. Acta Metall. 1973, 21, 571-574.

88. Brune, D.A.; Bicerano, J. Micromechanics of nanocomposites: comparison of tensile and compressive elastic moduli, and prediction of effects of incomplete exfoliation and imperfect alignment on modulus. Polymer 2002, 43, 369-337.

89. Wang, J.; Pyrz, R. Prediction of the overall moduli of layered-silicate reinforced nanocomposites, I: Basic theory and formulas. Compos. Sci. Technol. 2004, 64, 925-934.

90. Wang, J.; Pyrz, R. Prediction of the overall moduli of layered-silicate reinforced nanocomposites, II: Analyses. Compos. Sci. Technol. 2004, 64, 935-944.

91. Shia, D.; Hui, C.Y.; Burnside, S.D.; Giannelis, E.P. An interface model for the prediction of Young's modulus of layered silicate elastomer nanocomposites. Polym. Compos. 1998, 19, 608-617.

92. Barber, A.H.; Cohen, S.R.; Wagner, H.D. Measurement of carbon nanotube-polymer interfacial strength. Appl. Phys. Lett. 2003, 82, 4140-4142.

93. Barber, A.H.; Cohen, S.R.; Kenig, S.; Wagner, H.D. Interfacial fracture energy measurements for multi-walled carbon nanotubes pulled from a polymer matrix. Compos. Sci. Technol. 2004, 64, 2283-2289.

94. Frankland, S.J.V.; Caglar, A.; Brenner, D.W.; Griebel, M. Molecular simulation of the influence of chemical cross-links on the shear strength of carbon nanotubepolymer interfaces. J. Phys. Chem. B 2002, 106, 3046-3048.

95. Huh, J.Y.; Manias, E. unpublished data.

96. Chassapis, C.S.; Petrou, J.K.; Petropoulos, J.H.; Theodorou, D.N. Analysis of computed trajectories of penetrant micromolecules in a simulated polymeric material. Macromolecules 1996, 29, 3615-3624.

97. Nielsen, L.E. Models for the permeability of filled polymer systems. J. Macromol. Sci. (Chem.) 1967, A1, 929-942.

98. Cussler, E.L.; Hughes, S.E.; Ward, W.J.; Aris, R. Barrier membranes. J. Membr. Sci. 1988, 38, 161-174.

99. Fredrickson, G.H.; Bicerano, J. Barrier properties of oriented disk composites. J. Chem. Phys. 1999, 110, 2181-2188.

100. Bharadwaj, R.K. Modeling the barrier properties of polymer-layered silicate nanocomposites. Macromolecules 2001, 34, 9189-9192. 